PART II

THE FLASH PHASE OF SOLAR FLARES 


\title{
IMPULSIVE (FLASH) PHASE OF SOLAR FLARES: \\ HARD X-RAY, MICROWAVE, EUV AND \\ OPTICAL OBSERVATIONS
}

\author{
S. R. KANE \\ Space Sciences Laboratory, University of California, Berkeley, Calif. 94720, U.S.A.
}

\begin{abstract}
Recent observations of impulsive hard X-ray, microwave, EUV and optical emissions during solar flares are briefly reviewed in order to deduce the characteristics of the impulsive (flash) phase phenomenon in small solar flares particularly from the point of view of the acceleration of electrons and their role in producing the various impulsive phase emissions. Observed and deduced characteristics of the various electromagnetic emission sources are summarized (Table II). The deduced characteristics of the electron acceleration process (Table III) indicate a process with high acceleration efficiency. The observations are found to be consistent with a model in which electrons are accelerated in a series of short pulses each lasting for $\$ 1 \mathrm{~s}$ and the accelerated electrons provide the energy necessary for all the observed electromagnetic emissions produced during the flash phase of small solar flares. Models of the impulsive phase emissions in which energetic electrons play a prominant role are examined and crucial tests to check the accuracy of these models are indicated (Table IV).
\end{abstract}

\section{Introduction}

It is well known that the apparent characteristics of a solar flare depend strongly on the frequency of the observed radiation as well as the spacial and time resolution of the instrument used. In addition there are large variations in these characteristics from one flare to another. However, certain broad characteristics of solar flares can still be deduced from the observed time variation of the electromagnetic radiation. A flare can, in general, be roughly divided into three phases: Precursor, Impulsive Phase and Gradual Phase. These phases are schematically illustrated in Figure 1. The precursor is a relatively small slow increase in the radiation indicating a possible ocurrence of the impulsive and/or gradual phase within the following $10 \mathrm{~min}$ or so. The impulsive phase, which has a duration of $\sim 100 \mathrm{~s}$, is characterized by the most rapid increase and decrease in the radiation flux during the lifetime of the flare. The radiation flux may have one or more maxima which may occur quasiperiodically. In the gradual phase the radiation flux continues to increase, reaches a maximum after the impulsive phase maximum, and then decreases to the pre-flare level. The total duration of the gradual phase is $\gtrsim 10 \mathrm{~min}$. It should be emphasized here that all the three phases do not necessarily occur in all flares and in a given flare a particular phase may not be detectable at certain radiation frequencies. However, it can be stated as a general rule that if impulsive phase occurs in a given flare, the gradual phase will almost certainly occur in that flare.

In this paper we will be primarily concerned with the impulsive phase. The principal characteristics of this phase is the non-thermal radiation, such as impulsive hard X-ray, microwave and type III radio emission, which indicates acceleration of particles, particularly electrons from $10 \mathrm{keV}$ to a few hundreds of $\mathrm{keV}$. In the past 


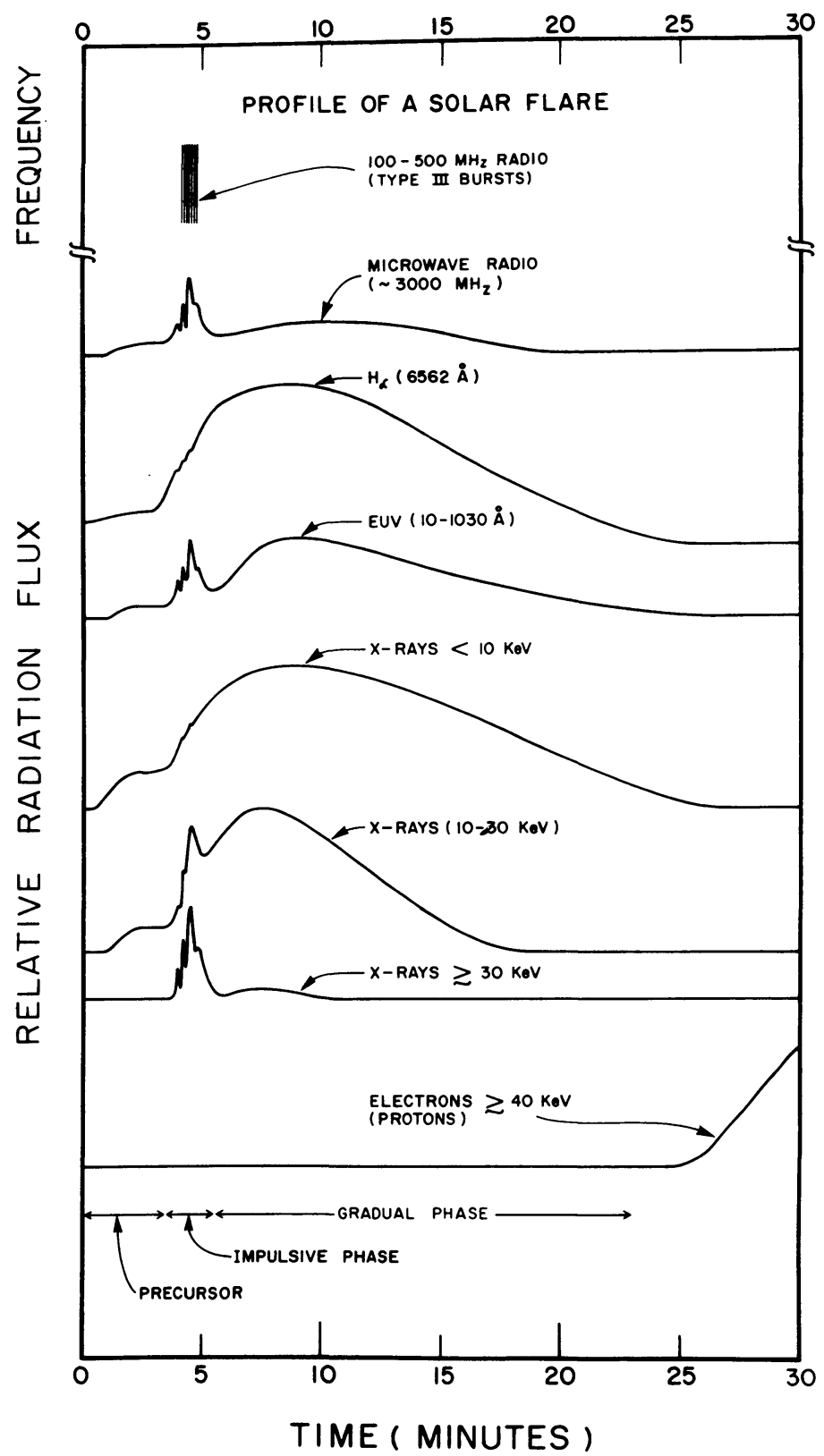

Fig. 1. A schematic representation of the different phases of a solar flare as observed in the electromagnetic and particle radiation.

this phase of the flare has been discussed in terms of two different phases observed in $\mathrm{H} \alpha$ emission: Flash Phase (Ellison, 1949) and Explosive Phase (Moreton, 1964). As we will find later in this paper, these two phases are probably a subgroup of the more general impulsive phase defined above. 
As a result of the work of Anderson and Winckler (1962), de Jager $(1964,1969)$, Kundu (1965), Moreton (1964), Peterson and Winckler (1959), Takakura and Kai (1966), Wild et al. (1963), and others, a conceptual picture of the flash phase or explosive phase had emerged in the early 1960's. It was recognized, for example, that acceleration of electrons was the most characteristic feature of the flash phase. Concepts regarding the propagation of electrons in the solar atmosphere and the associated hard X-ray, microwave and type III radio emission were also developed. What was lacking at that time was a quantitative picture. This was due to two reasons: (1) the number of well correlated observations, especially in the X-ray region, were small; (2) observations which could give quantities such as electron spectrum were not available.

With the launch of a simple hard X-ray detector aboard the OGO-1 satellite in 1964 (Arnoldy et al., 1967, 1968a, b, c) the number of correlated observations dramatically increased and have been steadily increasing since then. New phenomena such as EUV flares have been discovered and optical and radio observations with higher spacial and time resolution have been made. In this paper we briefly review these new observations and discuss their impact on the earlier concept of the flash phase. Since most of the new observations are related to flares of $\mathbf{H} \alpha$-importance $\lesssim 1$, the conclusions will be primarily applicable to small flares.

\section{Objectives of the Electromagnetic Radiation Measurements}

There are two principal objectives of the impulsive emission measurements from flares:

(1) To study the time variation of the emission from different parts of the flare region;

(2) To determine the location of the flare with respect to the magnetic field structure of the associated active region.

Both of these studies have important bearing on the physical mechanism of solar flares. It is not always possible to achieve both of the above objectives. For example, in the case of the hard X-ray emission, the spacial resolution of the detectors used so far was very poor and so only a part of the objective (1) could be fulfilled, viz. the study of the time variation of the $\mathrm{X}$-ray emission from the entire flare region. On the other hand, this difficulty is much less severe for the optical observations where spacial resolution of $\sim 0.5^{\prime \prime}$ is available with most of the recent instruments. In fact, the principal limitation of the optical measurements made so far has been the relatively poor time resolution $(\gtrsim 10 \mathrm{~s})$.

In general, the quantities of interest are the spectrum, directivity, polarization, spacial extent and altitude of the emission and the variation of these quantities with time. Since the impulsive phase is relatively short lived, measurements with highest available time resolution are to be emphasized. The characteristic time constants for the increase and decrease of the radiation flux at a given frequency are of particular interest because they indicate the rate of deposition and loss of non-thermal energy 
in that radiation source. This is of primary importance in determining the nature of the radiation process and the agency by which energy is transported from the point of primary energy release to the radiation source.

Since most of the recent observations are related to the flares of $\mathrm{H} \alpha$-importance $\lesssim 1$, the conclusions will be directly applicable only to relatively small flares as compared to the previous studies (Bruzek, 1967; de Jager, 1969) which were mainly related to large flares $(\mathrm{H} \alpha$-importance $\gtrsim 3)$. There are certain advantages in studying small flares. They occur more frequently and often have a relatively simple emission structure. Therefore the interpretation of observations is usually more reliable. Whenever possible, the small flare observations can of course be supplemented with the large flare observations so as to obtain a more general picture of the impulsive phase.

It has been apparent for some time that observations of one type of radiation, however extensive they may be, are not likely to reveal the true nature of the impulsive phase. Fortunately we now have a large number of simultaneous observations of impulsive X-ray, EUV, optical and radio emissions made during the past few years. The present problem of the impulsive phase is to construct a self-consistent picture based on the bits and pieces of information deduced from these different types of observations.

\section{X-Ray Observations}

Spectral observations of solar flare X-rays have been recently discussed by Neupert (1971), de Feiter (1972), Doschek (1972), and Kane (1973a). Although impulsive Xray emission may occasionally be detectable at X-ray energies of $\sim 5 \mathrm{keV}$ (Kahler and Kreplin, 1971; Peterson et al., 1973), in most flares it can be unambiguously measured only at energies $>10 \mathrm{keV}$. The observations of impulsive line emissions are scarce. In fact calculations of possible $\mathrm{K} \alpha$ line emission from $\mathrm{S}, \mathrm{Ar}, \mathrm{Ca}$ and $\mathrm{Fe}$ in the flare region show that the photon flux in these lines is expected to be weak during most impulsive events (Phillips and Neupert, 1973). We will therefore confine our discussion primarily to the impulsive continuum emission at $\mathrm{X}$-ray energies $\gtrsim 10 \mathrm{keV}$.

The early hard X-ray measurements were made with balloons (Peterson and Winckler, 1959; Vette and Casal, 1961; Anderson and Winckler, 1962). These experiments suffered from short observation time $(\sim 24 \mathrm{~h}$ per flight) and the effects of the Earth's atmosphere on the incident solar X-ray spectrum. However, such measurements gave rise to the discovery of hard solar X-ray bursts and even now new significant results are being obtained with balloon borne detectors (Parks and Winckler, 1969; Takakura et al., 1971; Kane et al., 1972). The rocket measurements were relatively free from atmospheric effects and hence the X-ray spectrum could be accurately measured down to a few $\mathrm{keV}$ (Chubb et al., 1966). However, the problem of the short observation time (a few minutes) was even more severe in this type of measurement. Consequently only a few hard X-ray bursts from rather large solar flares were observed with balloons and rockets during the period 1958-1964.

With the advent of the satellites the difficulties due to the short observation time 
and effects of the Earth's atmosphere were simultaneously removed. Since 1964 a large number of hard X-ray bursts have been recorded with the ionization chambers aboard the OGO-1 and OGO-3 satellites (Arnoldy et al., 1968a, b, c; Kane and Winckler, 1969a, b) and the scintillation spectrometers aboard the following satellites: OSO-3 and OSO-7 (Hudson et al., 1969; Elcan, 1973; Peterson et al., 1973), OGO-3 (Cline et al., 1968), OGO-5 (Kane and Anderson, 1970; Kane, 1971), and OSO-5 (Frost, 1969; Frost and Dennis, 1971). In addition, polarization of 10-20 keV X-rays has been measured with the Intercosmos -1 and 4 spacecraft (Tindo et al., 1970, 1972a; Mandelstam, 1972).

Figure 2 shows an example of a relatively simple impulsive X-ray burst observed by the OGO-5 satellite (Kane and Anderson, 1970). The associated microwave emission is also shown for comparison. The impulsive $\mathrm{X}$-ray emission, which was most prominent at X-ray energies $\gtrsim 20 \mathrm{keV}$, reached its maximum simultaneously with the maximum of the impulsive microwave emission but $\sim 1.5 \mathrm{~min}$ before the maximum of the $\mathrm{H} \alpha$ emission from the total flare area. Sometimes the X-ray bursts consist of several impulsive peaks which may occur quasi-periodically with a period of 10-30 s (Parks and Winckler, 1969, 1971; Frost, 1969).

In X-ray bursts such as the one shown in Figure 2 one can identify two X-ray components: (1) an impulsive component which is more prominent at $\mathrm{X}$-ray energies $\gtrsim 30 \mathrm{keV}$ and may exhibit quasi-periodic fluctuations; (2) a gradual component with a relatively smooth time-intensity profile and which reaches its maximum several minutes after the maximum of the impulsive component. The existence of two components in the $\mathrm{X}$-ray emission $\gtrsim 10 \mathrm{keV}$ was suggested earlier by several workers (Acton, 1968; Takakura, 1969; de Jager, 1969). The two component structure was first observed in the OGO-5 data (Kane, 1969; Kane and Anderson, 1970) had has since then been found to be consistent with the OSO-3, OSO-5 and OSO-7 measurements.

The X-ray spectrum is related to the spectrum of the energetic electrons in the $\mathrm{X}$-ray source. For example, a power law X-ray spectrum $\sim E^{-\gamma}$ photons $\mathrm{cm}^{-2} \mathrm{~s}^{-1}$ $\mathrm{keV}^{-1}$ indicates a power law electron spectrum $\sim E_{e}^{-\alpha}$ electrons $\mathrm{keV}^{-1}$ where $\gamma-\alpha \approx 1$ (Kane and Anderson, 1970; Brown, 1971). It is therefore important to have accurate high time resolution measurements of the $\mathrm{X}$-ray spectrum so that the characteristics of the electron spectrum can be deduced. Figure 3 shows the X-ray spectrum in 10-100 keV range measured at the maxima of three impulsive X-ray bursts observed by the OGO-5 satellite (Kane, 1973a). A spectrum measurement of $10-200 \mathrm{keV}$ $\mathrm{X}$-rays made with the OSO-5 satellite has been reported by Frost (1969) and Frost and Dennis (1971)(Figure 9). For X-rays in the energy range $E_{\mathrm{C} 1}-E_{\mathrm{C} 2}$, the observed spectrum is consistent with the form

$$
\frac{\mathrm{d} J}{\mathrm{~d} E}=K E^{-\gamma} \text { photons } \mathrm{cm}^{-2} \mathrm{~s}^{-1} \mathrm{keV}^{-1} .
$$

Above some critical energy $E_{\mathrm{C} 2}$ the spectrum steepens rapidly (larger $\gamma$ ). The value of $E_{\mathrm{C} 2}$ varies from one flare to another but usually lies in the range $60-100 \mathrm{keV}$. A large 


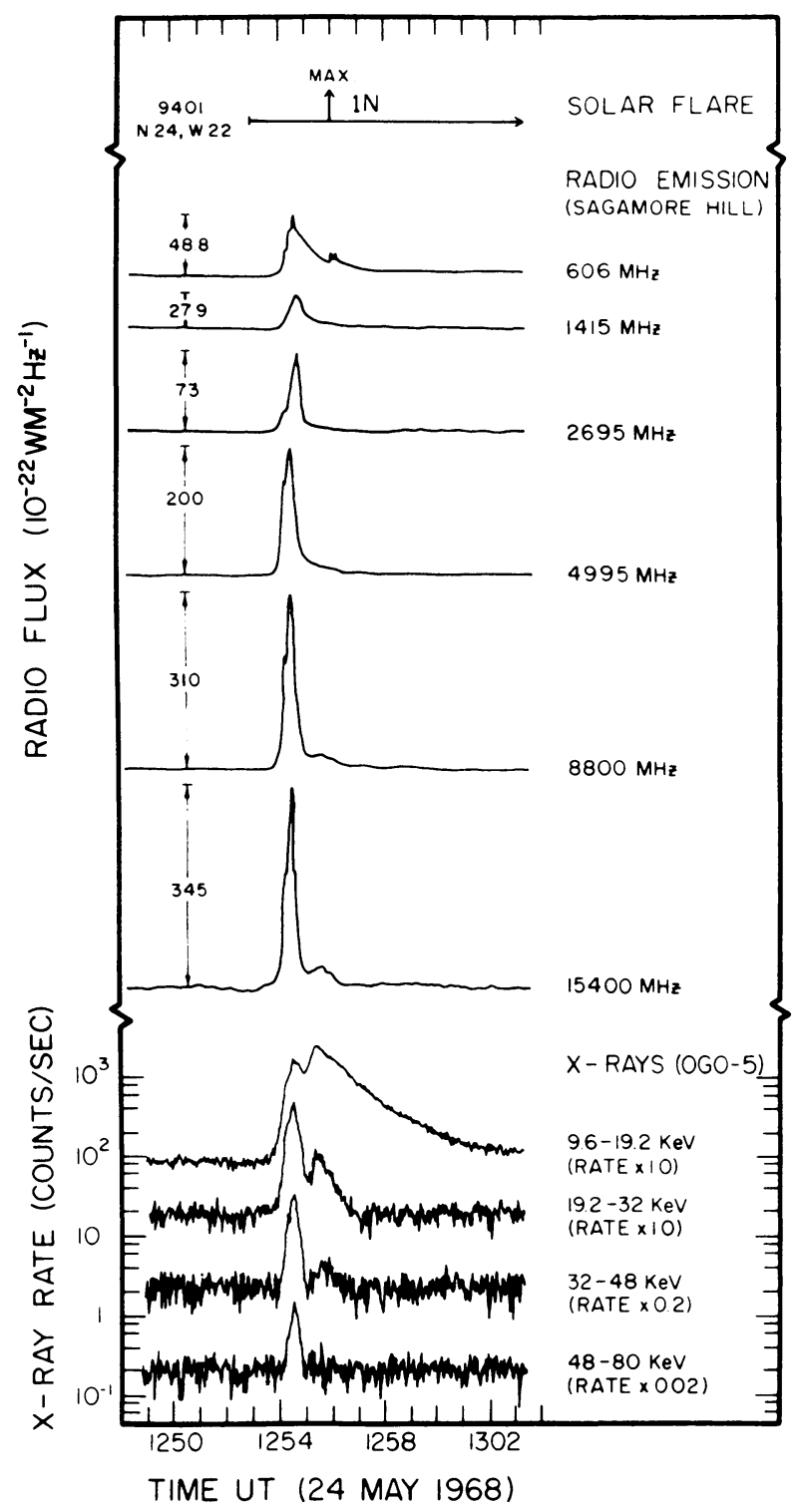

Fig. 2. An example of a relatively simple impulsive $\mathrm{X}$-ray and microwave burst (Kane and Anderson, 1970).

flare in which $E_{\mathrm{C} 2}$ might have been $\sim 500 \mathrm{keV}$ has been reported by Gruber et al. (1973). Below some critical energy $E_{\mathrm{C} 1}$ the gradual X-ray component dominates and the impulsive X-ray spectrum cannot be determined un-ambiguously. Usually $E_{\mathrm{C} 1}$ is $\sim 20 \mathrm{keV}$. However, occasionally it may be as low as $\sim 5 \mathrm{keV}$ as was observed by Kahler and Kreplin (1971), and Peterson et al. (1973) (Figure 4).

The spectral exponent $\gamma$ also varies from one flare to another. Figure 5 shows the 


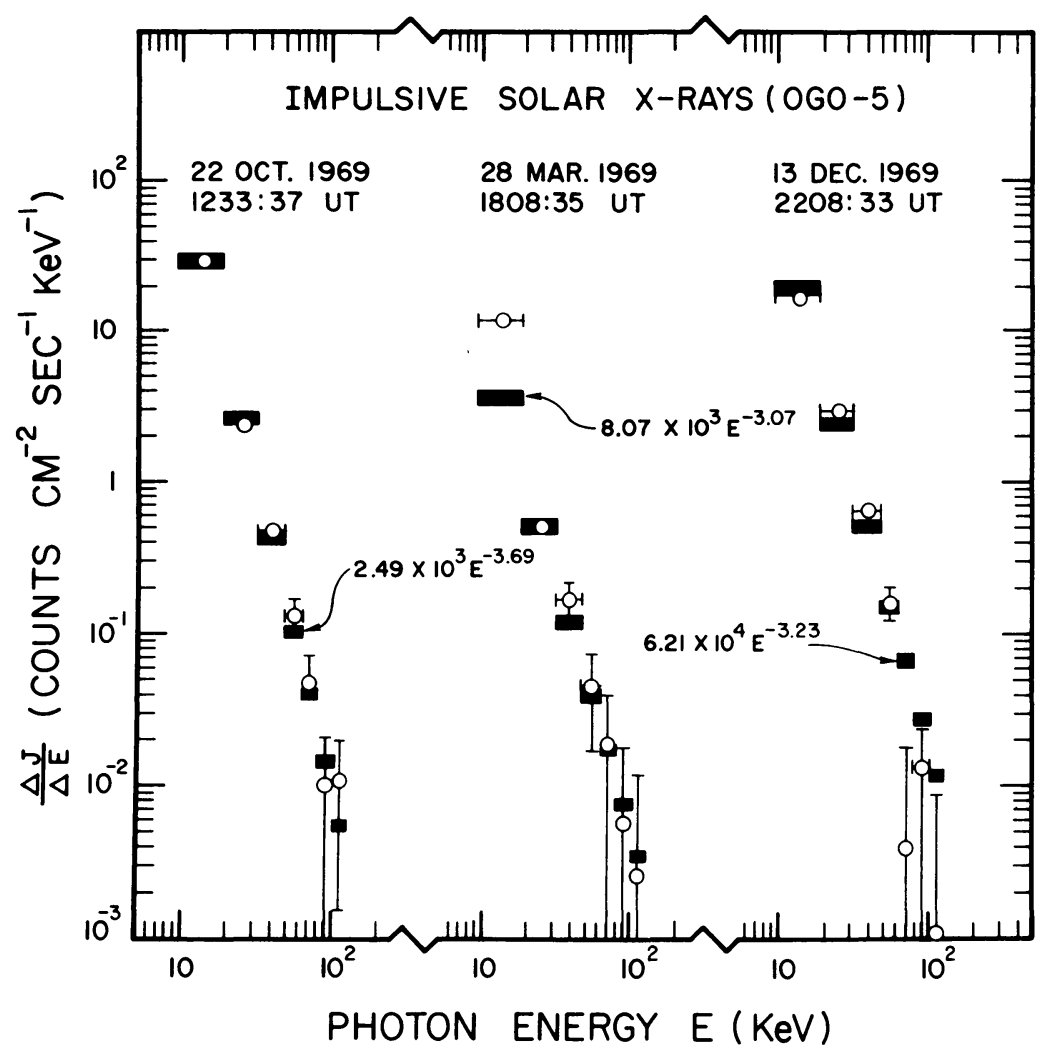

Fig. 3. The X-ray spectra at the maxima of three impulsive bursts. Open circles are observations and dark rectangles are computed response of the OGO-5 detector to a power law X-ray spectrum (Kane, 1973a).

frequency of occurrence of different values of $\gamma$ in small solar flares (Kane, 1973a). For $2.5 \lesssim \gamma \lesssim 5.0$ the probability of occurrence of a given $\gamma$ in a flare increases systematically with the increase in the value of $\gamma$. The probability of occurrence of a flare with $\gamma<2.5$ is extremely small indicating that there is essentially an upper limit on the hardness of the impulsive X-ray spectrum (and hence the electron spectrum) produced in solar flares (Kane, 1971).

The time variation of the impulsive X-ray spectrum during a single flare is of particular interest because it is expected to give information about the acceleration and energy loss processes for the energetic electrons in the flare region. At present there is no general agreement on the nature of this variation. In several small X-ray bursts Kane and Anderson (1970) find that the X-ray spectrum in 10-60 keV range hardens from the onset of the X-ray burst to its maximum and then softens during the decay (Table I). Similar spectral variation has been observed by Parks and Winckler (1969) and Kodama et al. (1971) in a large X-ray burst recorded with balloon born detectors. The softening of the X-ray spectrum during the decay has also been reported by McKenzie et al. (1973) and Peterson et al. (1973). One case of 
hardening of the spectrum during the increasing phase of the X-ray burst has been observed by Peterson et al. (1973). In some events no systematic variation of the spectrum is observed during the increasing phase (McKenzie et al., 1973; Datlowe and Peterson, 1973). Frost (1969) and Frost and Dennis (1971) have each reported one burst associated with a large flare in which the X-ray spectral exponent was constant throughout the duration of the $\mathrm{X}$-ray burst. On the other hand, for one large flare, Cline et al. (1968) found that the X-ray spectrum $\gtrsim 80 \mathrm{keV}$ hardened during the decay phase. A similar effect has been reported by Gruber et al. (1973) for the 23 May 1967 flare events. The discrepancy between these different observations could be due to the inherent variations from one flare to another. Another factor could be the fine time structure $(\lesssim 1 \mathrm{~s})$ in the X-ray time-intensity profile and the associated spectral variations. Clearly, high time resolution ( $\sim 0.1 \mathrm{~s})$ measurements of a large number of flares are required to resolve this question satisfactorily.

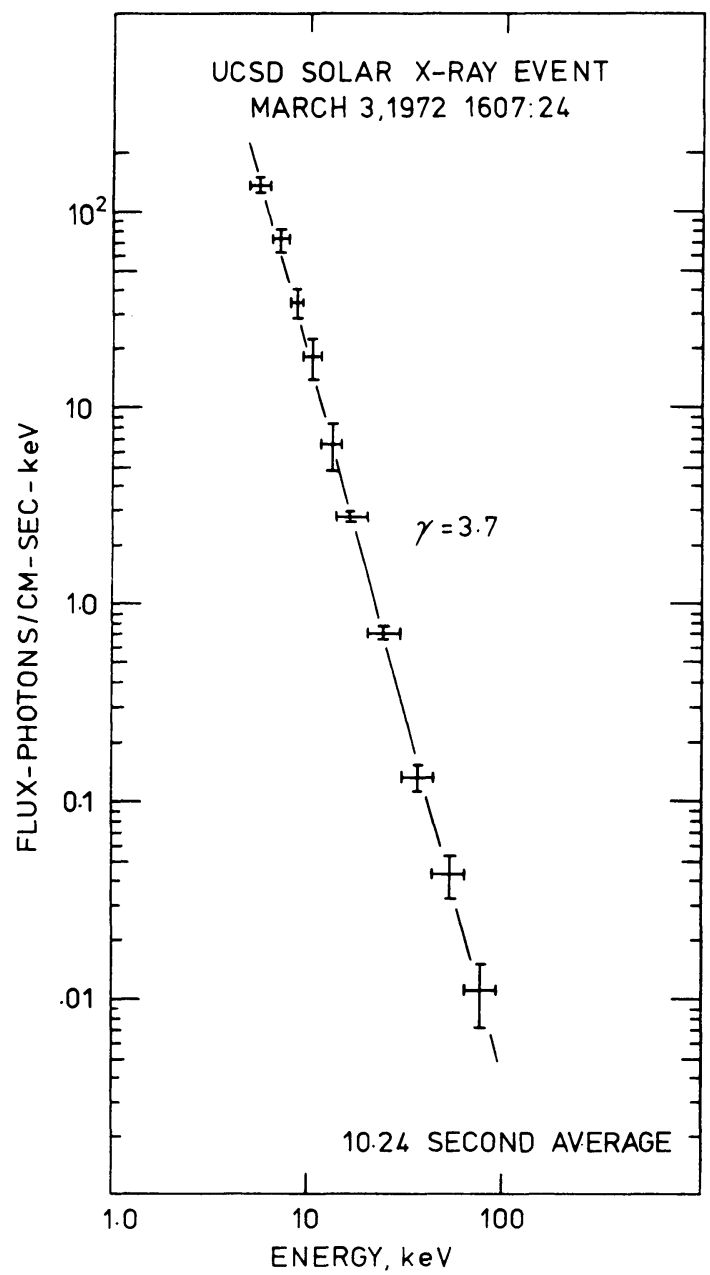

Fig. 4. An example of the power law X-ray spectrum extending down to $\sim 5 \mathrm{keV}$ (Peterson et al., 1973). 


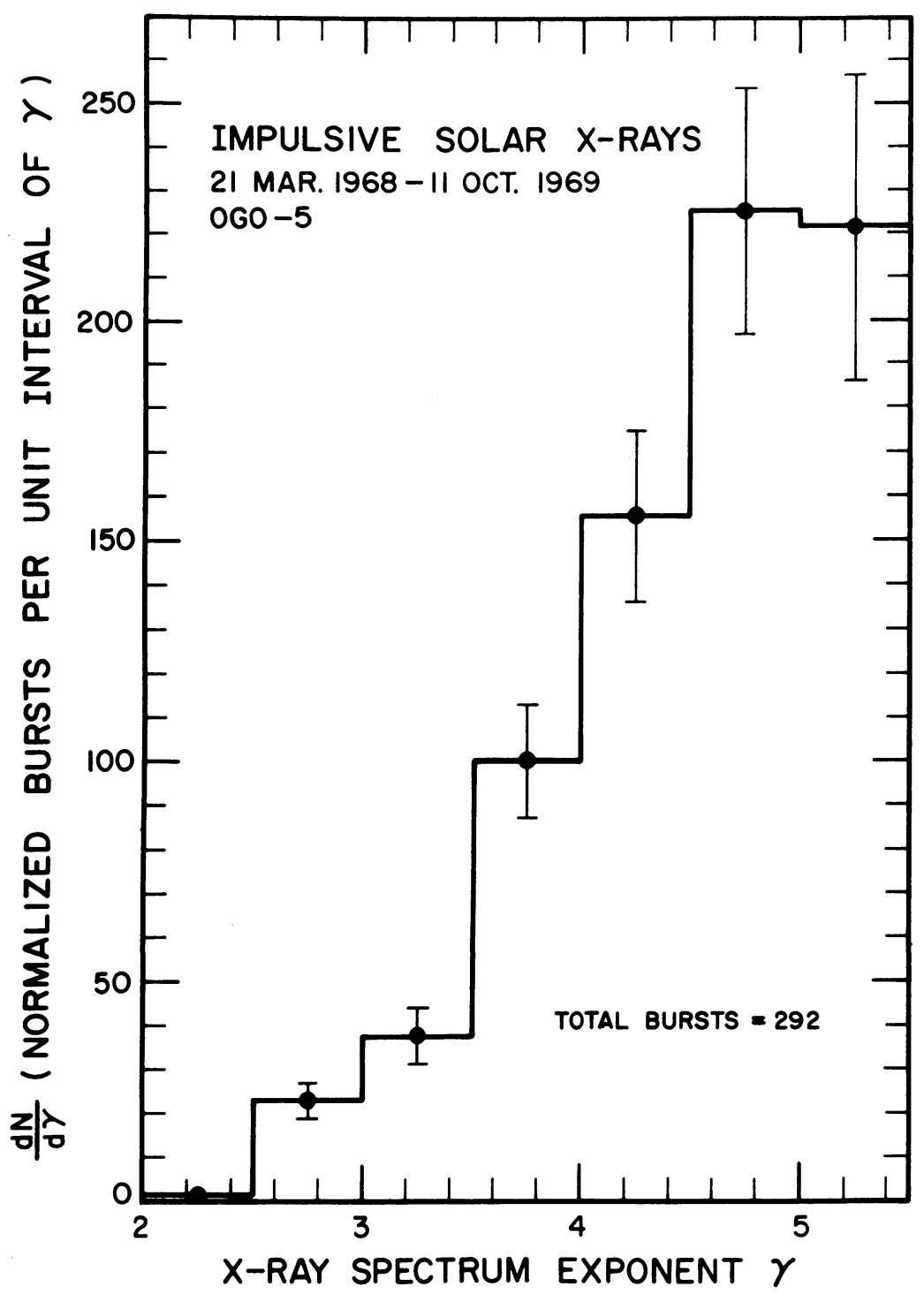

Fig. 5. The relative frequency of occurrence of the different values of the X-ray spectral exponent $\gamma$ in small solar flares (Kane, 1973a).

TABLE I

Impulsive $\mathrm{X}$-rays $(\gtrsim 10 \mathrm{keV})$ from small solar flares

\begin{tabular}{|c|c|}
\hline Spectrum at maximum (at $1 \mathrm{AU}$ ) & $\begin{array}{l}\sim E^{-\gamma} \text { photons } \mathrm{cm}^{-2} \mathrm{~s}^{-1} \mathrm{keV}^{-1} \\
2<\gamma \leqslant 6 \text { for } 10 \lessgtr E \$ 100 \mathrm{keV} \\
\text { Much steeper for } E>100 \mathrm{keV}\end{array}$ \\
\hline Maximum energy flux at $1 \mathrm{AU}$ & $10^{-7}-10^{-5} \mathrm{erg} \mathrm{cm}^{-2} \mathrm{~s}^{-1}$ for $10 \leqslant E \leqslant 100 \mathrm{keV}$ \\
\hline Rise time & $2-5 \mathrm{~s}$ for $E \sim 40 \mathrm{keV}$ less for $E>40 \mathrm{keV}$ \\
\hline Decay time ( $\gtrsim$ rise time) & 3-10 s for $E \sim 40 \mathrm{keV}$ less for $E>40 \mathrm{keV}$ \\
\hline Correlated emissions & Impulsive microwave, EUV, $\mathrm{H} \alpha$ type III radio \\
\hline
\end{tabular}


A question of some interest is the variation of the peak flux and the spectral exponent $\gamma$ with the location of the associated $\mathrm{H} \alpha$-flare on the solar disc. Since the absorption of hard X-rays in the solar atmosphere is negligible, any variation in the characteristics or number of the X-ray bursts with the location of the flare may be an indication of the directivity of the X-ray emission. Since large variations in the burst characteristics are observed even for flares having essentially the same location on the disc, it is essential to analyze a large number of events before a statistically significant result can be obtained. Two somewhat different results have been reported so far. From an analysis of 46 hard X-ray burst recorded by the OGO-1 and OGO-3 ionization chambers (Arnoldy et al., 1968c) and the OSO-1 spectrometer (Frost, 1964), Ohki (1969) has concluded that the number of X-ray bursts decreases systematically from the center to the limb of the solar disc indicating a limb-darkening. On the other hand, using similar data Pinter (1969) found that the number distribution of the X-ray bursts has two maxima, one at $20-30^{\circ} \mathrm{E}$ and the other at $20-30^{\circ} \mathrm{W}$ heliographic longitude. The difference between these two results is most probably due to the statistical uncertainties caused by the relatively small number of events used in the analysis. Figure 6 shows the number distribution obtained for $\sim 300$ impulsive X-ray bursts observed with the OGO-5 satellite. All of these bursts were associated with

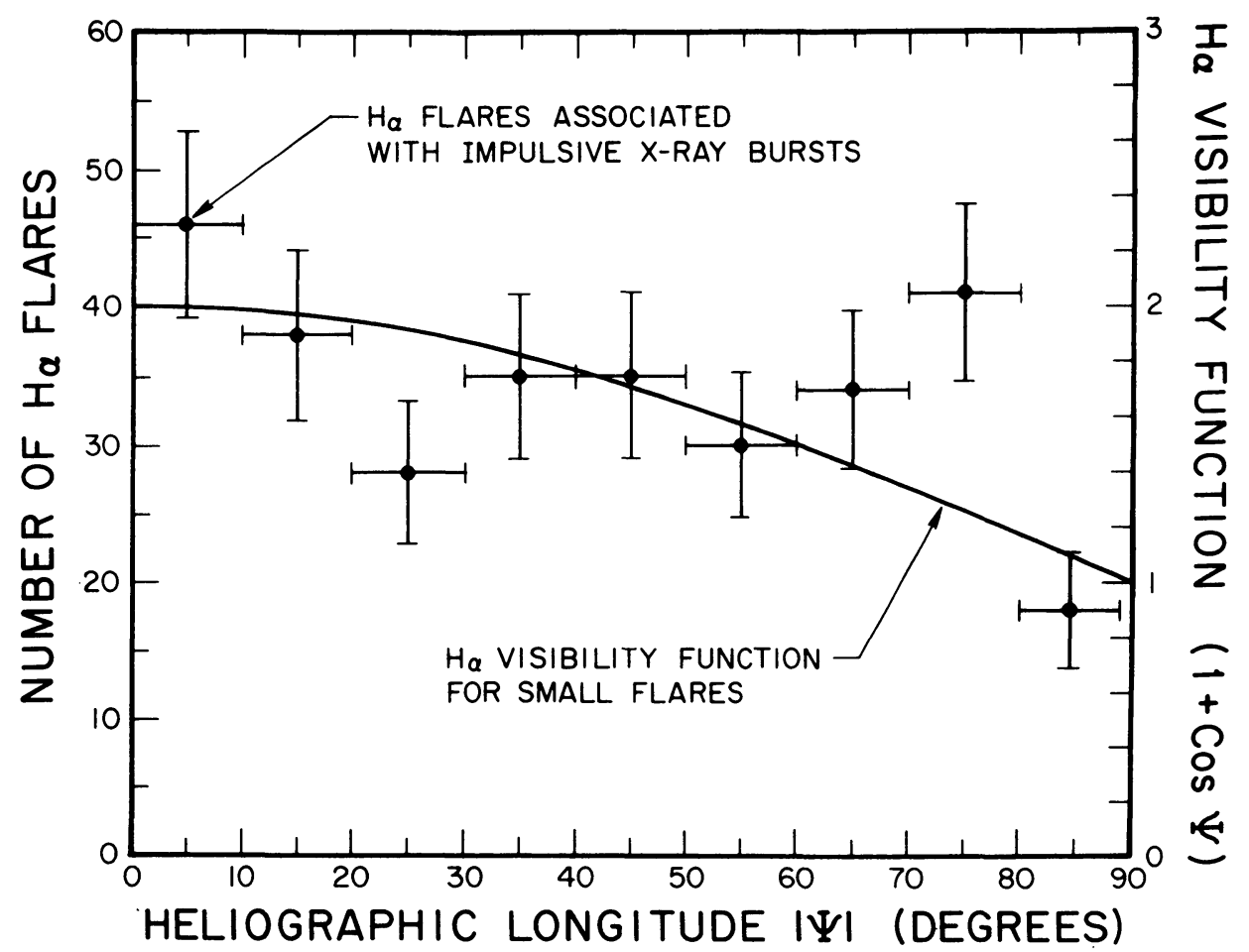

Fig. 6. Center-to-limb variation of the location of the $\mathrm{H} \alpha$-flares associated with $\sim 300$ impulsive $\mathrm{X}$-ray bursts observed by the OGO-5 satellite. The solid line represents the $\mathrm{H} \alpha$ visibility function for small flares given by Smith and Smith (1963). 
flares of $\mathrm{H} \alpha$-importance $\lesssim 1$. Therefore the ' $\mathrm{H} \alpha$ visibility function' for small solar flares (Smith and Smith, 1963) is also shown in Figure 6 for comparison. It can be seen that at least in the case of small flares there is no significant center-to-limb variation in the frequency of occurrence of X-ray bursts.

Another indication of the directivity of the X-ray emission and/or the directivity of the energetic electrons producing the observed X-ray emission is its polarization. The linear polarization of 10-20 keV X-rays in several solar flares has been measured with the Intercosmos-1 and 4 spacecraft (Tindo et al., 1970, 1972a, b; Mandelstam, 1972). An example taken from Tindo et al. (1972a) is shown in Figure 7. Here the three curves (a), (b) and (c) respectively represent the intensity of the radiation, degree of polarization and the angle of polarization (angle between the plane of polarization and the 'reference' plane of the polarimeter). The measurements have been normalized in such a way that the polarization is zero during the late decay phase of the gradual $\mathrm{X}$-ray burst. The authors have found significant polarization for time intervals lasting from a few up to ten minutes. At the maximum of the impulsive burst the polarization is $\sim 20 \%$ and the plane of polarization is so oriented that its projection on the solar disc passes through the associated flare region and the denter of the disc. An increase in polarization is also found at the maximum of the gradual X-ray component.

Direct measurements of the location and size of the impulsive hard X-ray source are difficult because of the high time resolution necessary to obtain meaningful results. One dimensional measurements of one X-ray burst has been made by Takakura et al. (1971) with a balloon born modulation collimator. They found the center of the $\mathrm{X}$-ray source to be on a line passing through the center of the associated $\mathrm{H} \alpha$ flare (Figure 8). Although the $\mathrm{H} \alpha$ flare region was $\sim 3^{\prime}$, the size of the hard X-ray source was $\lesssim 1^{\prime}$.

At present the only source of information about the altitude of the impulsive hard $\mathrm{X}$-ray source are the behind-the-limb flares. These are invariably relatively large flares and the information is therefore directly applicable only to large flares. Figure 9 shows an impulsive X-ray burst from a flare $\sim 14^{\circ}$ behind the west limb (Frost and Dennis, 1971). It shows that the source of the observed emission was located at an altitude $>10^{4} \mathrm{~km}$ above the photosphere. McKenzie and Peterson (1973) have recently reported observations of five behind-the-limb flares which are consistent with such a conclusion for small flares.

It should be pointed out here that $\mathrm{X}$-ray emission produced at the flare site is subject to Compton scattering in the solar atmosphere. The X-rays observed at $1 \mathrm{AU}$ therefore consist of the relatively unaffected direct beam of photons as well as the Compton back-scattered photons. Calculations show that the relative flux of the direct and scattered photons depends on the inial energy of the photons and the location of the X-ray source on the sun (Tomblin, 1972; Santangelo et al., 1973). At present it appears that the corrections due to this 'albedo effect' are of the same order of magnitude as the uncertainties in the calculations themselves and the uncertainties in the measured parameters, such as spectral index and polarization, caused by statistical fluctuations and the limited spectral, temporal and spatial 


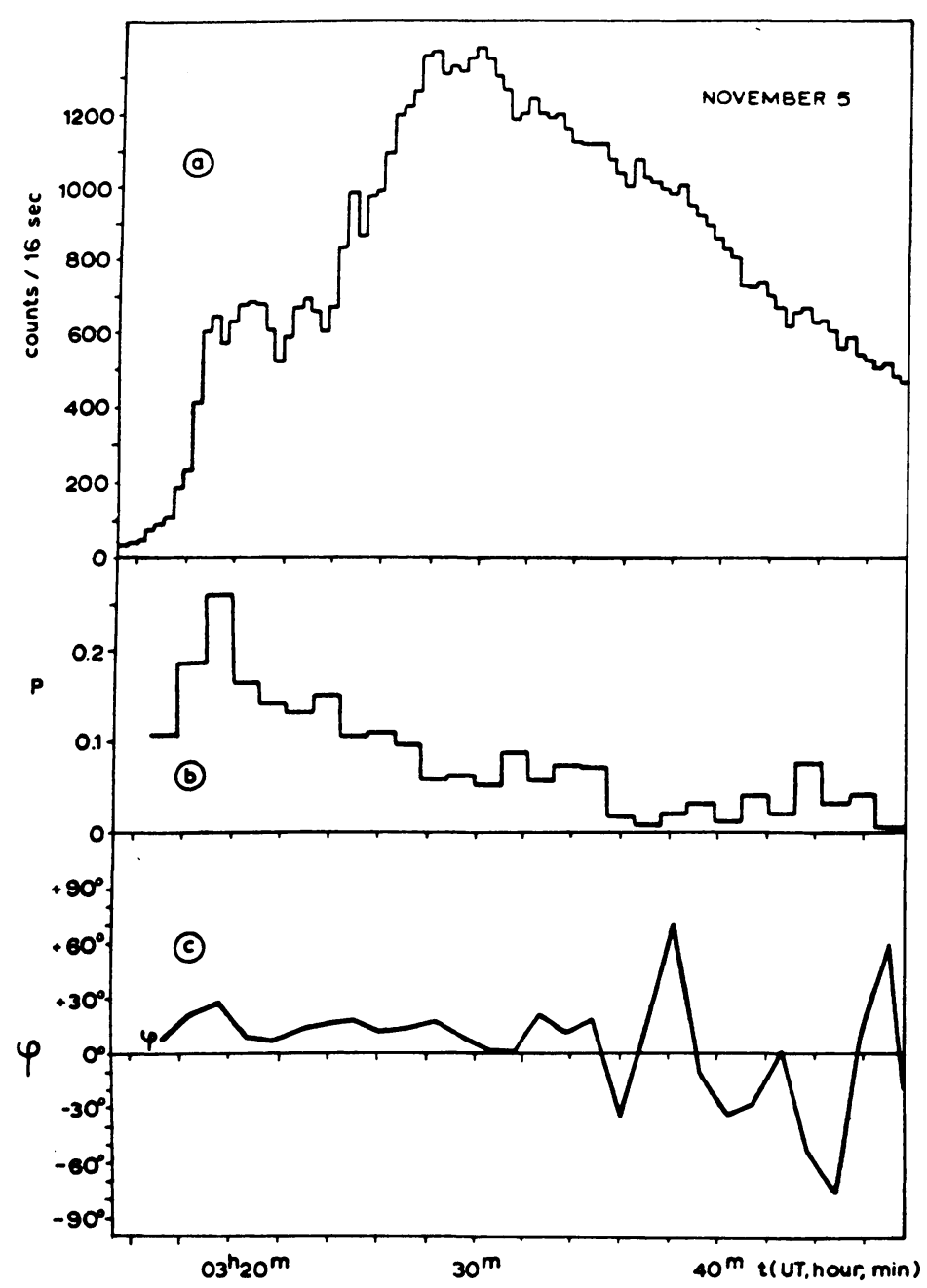

Fig. 7. Linear polarization of 10-20 keV X-rays measured with the Intercosmos 4 spacecraft. The three curves are: (a) intensity of the radiation, (b) degree of polarization, and (c) angle of polarization (Tindo et al., 1972a).

resolution of the instruments. Therefore not much is likely to be gained by applying corrections for the albedo effect to the presently available observations. However as the spatial, temporal and spectral resolution of the instruments improves in the future, the albedo effect must be taken into account.

\section{Radio Observations}

The observations of flare associated radio emission have been widely discussed in the past (Kundu, 1965; Maxwell, 1965; Takakura, 1969; Boischot, 1972). The radio bursts most intimately associated with the impulsive phase of flares are the impulsive 


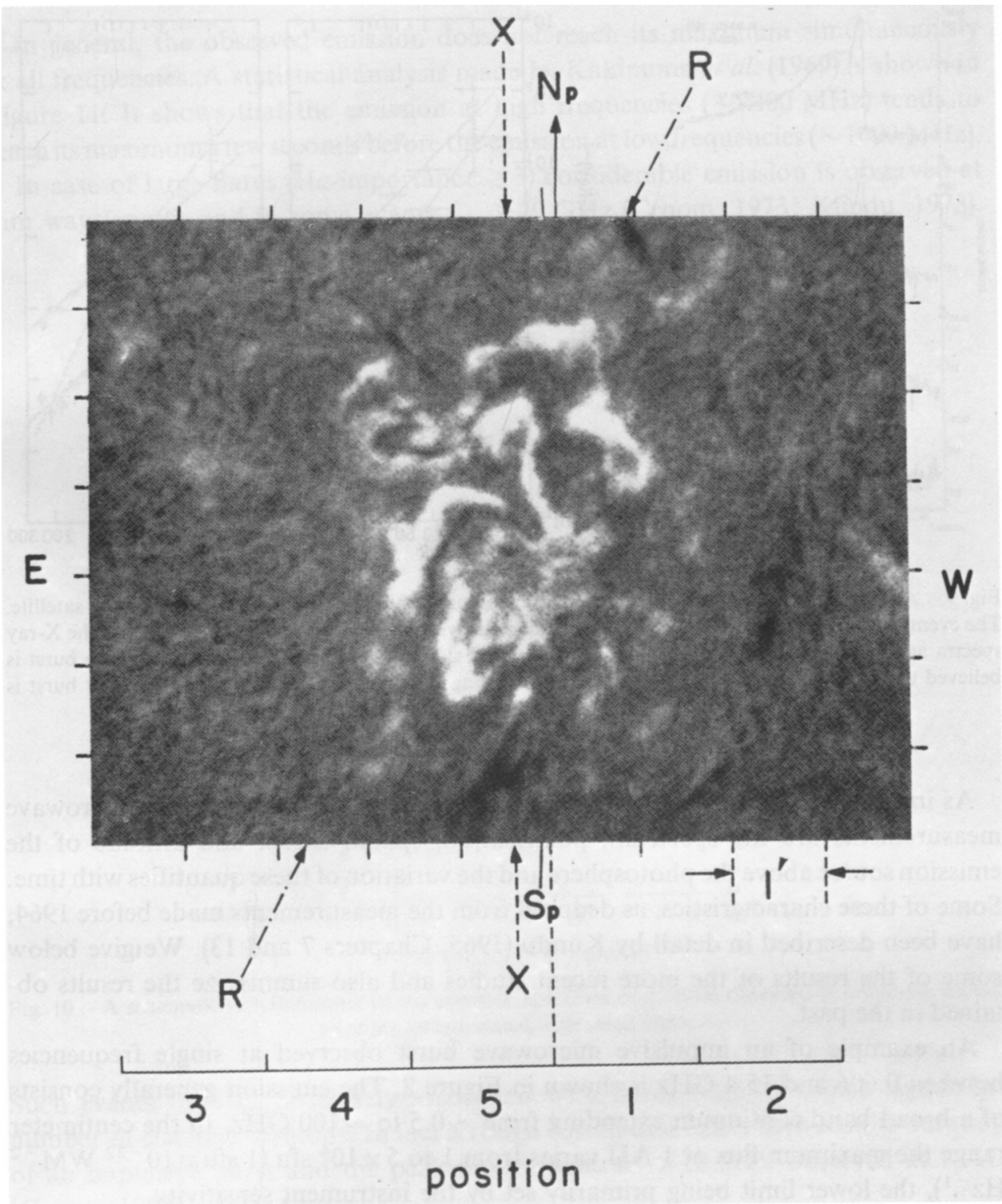

Fig. 8. Location of the centers of the hard X-ray source (line $\mathrm{X}$ ) and $3750 \mathrm{MHz}$ source (line R) with respect to the associated $\mathrm{H} \alpha$-flare region (Takakura et al., 1971).

microwave bursts ( $\lambda \lesssim 10 \mathrm{~cm}, f \gtrsim 3 \mathrm{GHz}$ ) and the type III radio bursts at decimeter and meter wavelengths. In this section we will be mostly concerned with the impulsive microwave bursts. The ground based and satellite observations of the type III bursts are discussed elsewhere in the proceedings of this symposium (Stewart, 1973; Fainberg, 1973). Therefore we will only mention here the recent correlation studies related to the type III bursts, particularly those extending into the decimeter range. 

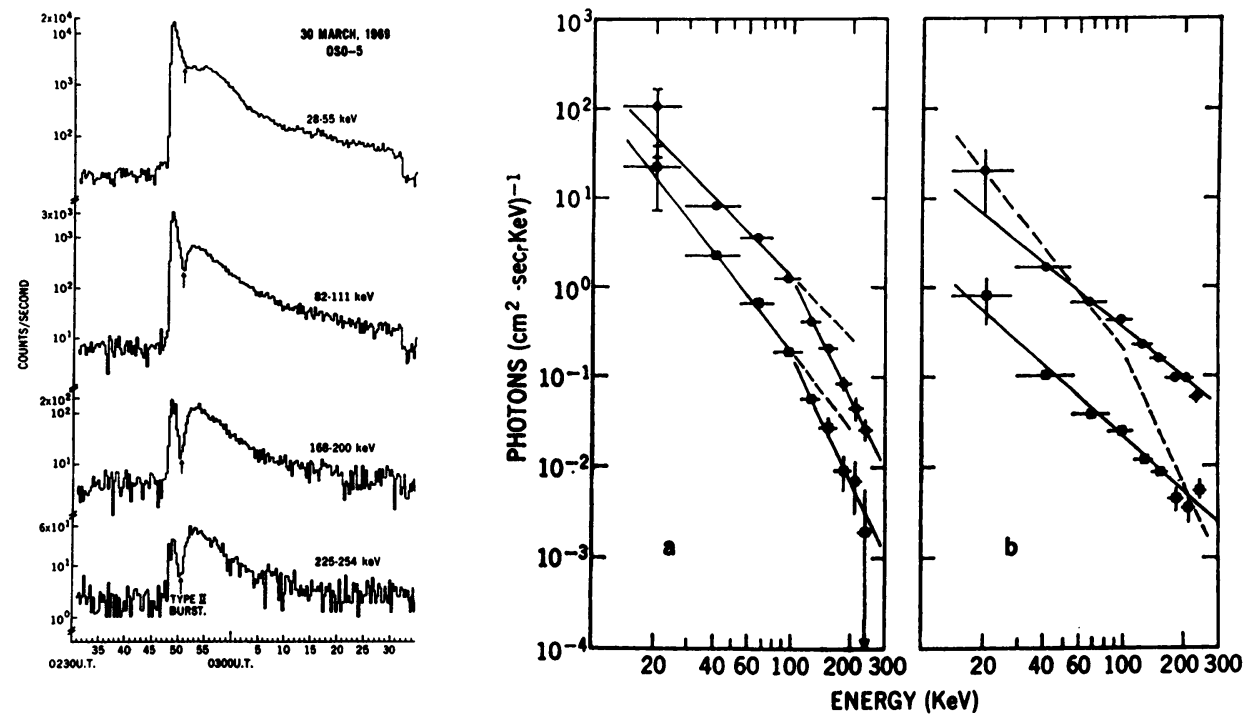

Fig. 9. An impulsive $\mathrm{X}$-ray burst followed by a slower long lasting burst observed by the OSO-5 satellite. The event was associated with a flare located behind the west limb of the Sun. (a) and (b) show the X-ray spectra at two different times during the impulsive and slow bursts respectively. The impulsive burst is believed to be caused by acceleration of electrons during the impulsive (flash) phase. The slow burst is considered evidence for a second stage of acceleration (Frost and Dennis, 1971).

As in the case of X-ray measurements the quantities of interest in the microwave measurements are the spectrum, polarization, spatial extent and altitude of the emission source above the photosphere and the variation of these quantities with time. Some of these characteristics, as deduced from the measurements made before 1964, have been described in detail by Kundu (1965, Chapters 7 and 13). We give below some of the results of the more recent studies and also summarize the results obtained in the past.

An example of an impulsive microwave burst observed at single frequencies between 0.606 and $15.4 \mathrm{GHz}$ is shown in Figure 2. The emission generally consists of a broad band continuum extending from $\sim 0.5$ to $\sim 100 \mathrm{GHz}$. In the centimeter range the maximum flux at $1 \mathrm{AU}$ varies from 1 to $5 \times 10^{4} \mathrm{sfu}\left(1 \mathrm{sfu} \equiv 10^{-22} \mathrm{WM}^{-2}\right.$ $\mathrm{Hz}^{-1}$ ), the lower limit being primarily set by the instrument sensitivity.

The spectra of the impulsive microwave bursts have been recently compiled by Guidice and Castelli (1973) and Croom (1973). The spectrum most often associated with small and medium flares (H $\alpha$-importance $\$ 2)$ is what Guidice and Castelli $(1973)$ have called type C. It is schematically shown in Figure 10 . This is a relatively simple spectrum in which the flux reaches a single maximum at a frequency $f_{\max }$ in the centimeter range and decreases on both sides of this maximum. The frequency of maximum emission $f_{\max }$ is usually $\sim 5 \mathrm{GHz}$ and the emission at $\mathrm{mm}$ wavelengths $(f>10 \mathrm{GHz})$ is $<50 \%$ of the emission at $f_{\max }$. At the low frequency side the spectrum has an effective cut-off at a frequency $f_{\text {cut }} \approx 0.3 f_{\max }$ (Guidice and Castelli, 1973). 
In general, the observed emission does not reach its maximum simultaneously at all frequencies. A statistical analysis made by Kakinuma et al. (1969) is shown in Figure 11. It shows that the emission at high frequencies $(\sim 9400 \mathrm{MHz})$ tends to reach its maximum a few seconds before the emission at low frequencies $(\sim 1000 \mathrm{MHz})$.

In case of large flares $(\mathrm{H} \alpha$-importance $\gtrsim 3)$ considerable emission is observed at $\mathrm{mm}$ wavelengths and in some events $f_{\max } \gtrsim 20 \mathrm{GHz}$ (Croom, 1973; Kundu, 1973).

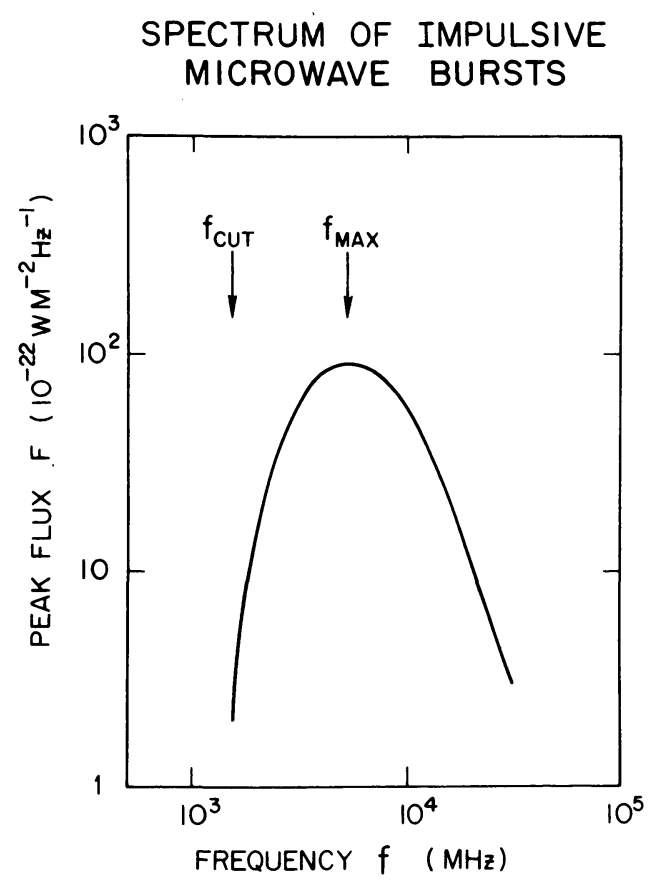

Fig. 10. A schematic representation of the emission spectrum most often observed in impulsive microwave bursts associated with small flares.

Such events tend to be closely associated with flares which produce significant number of energetic protons. In fact a rough correlation exists between the duration of an impulsive burst and the peak flux of protons $>10 \mathrm{MeV}$ observed at $1 \mathrm{AU}$ (Croom, 1973).

High resolution measurements at $9.4 \mathrm{GHz}$ by Tanaka et al. (1967) show that some impulsive bursts consist of two different emission sources separated by $\sim 2^{\prime}$ and coinciding with the two sunspots of a bipolar group. Both sources are circularly polarized but in opposite directions. Figure 12 shows the time development of the intensity and polarization distribution observed at $9.4 \mathrm{GHz}$ by Enome et al. (1969) during the 16 December 1967 flare event. A hard X-ray burst was also observed in this event (Kane and Winckler, 1969b) approximately in time coincidence with the impulsive microwave burst which reached its maximum at 0252 UT. Initially (02460249 UT) and after the flux maximum (0255-0258 UT) the right hand polarization $(R)$ 
was dominant in the whole radio source. However, near the burst maximum(0252 UT) the left handed polarization $(L)$ dominated in the eastern part of the source. This change in polarization during the increasing phase of the burst, for which no optical counterpart was found in $\mathrm{H} \alpha$ photographs, was observed at $9.4 \mathrm{GHz}$ but not at $3.75 \mathrm{GHz}$. The authors have interpreted this observation as indirect evidence for a
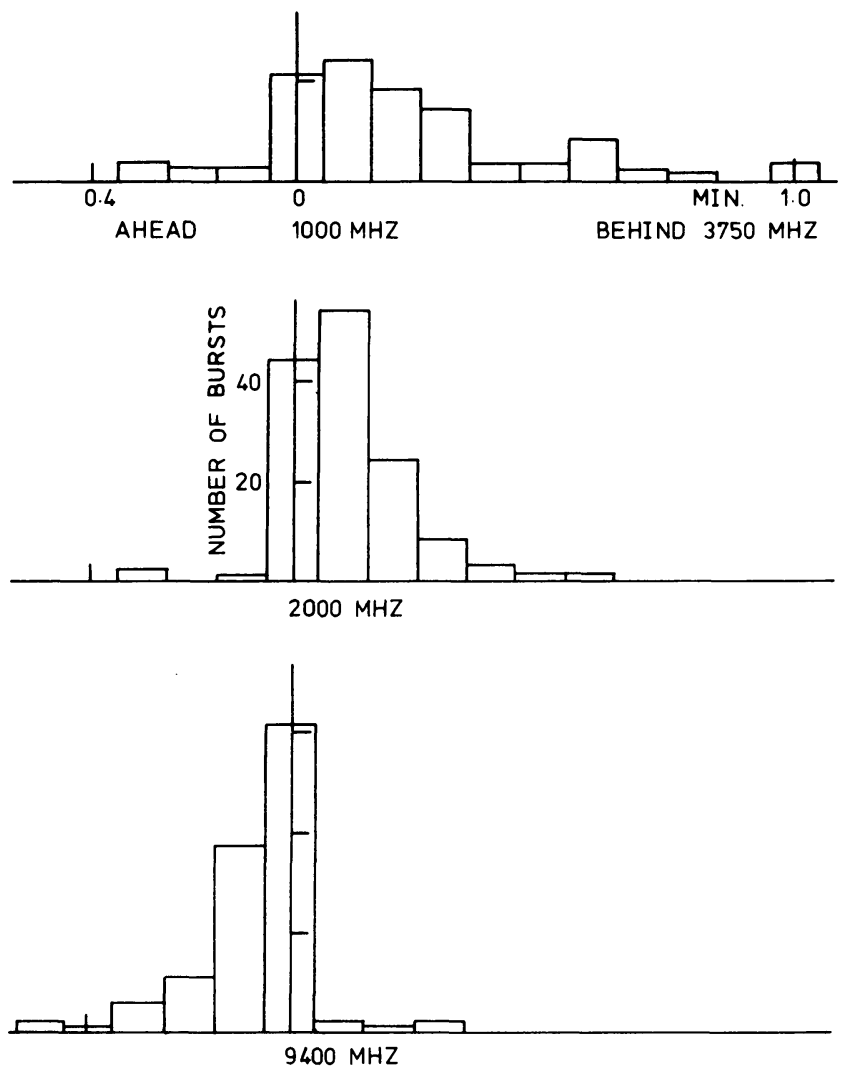

Fig. 11. A statistical analysis of the differences in the times of maximum intensity 1000,2000 and 9400 $\mathrm{MHz}$ with respect to the time of maximum at $3750 \mathrm{MHz}$. On an average, the emission at 1000 and 2000 MHz tends to reach its maximum later than the emission at 9400 and $3750 \mathrm{MHz}$ (Kakinuma et al., 1969).

change in the magnetic field configuration of the associated active region during the impulsive burst.

The center-to-limb variation of the characteristics of the microwave bursts have been studied by several workers (Dodson et al., 1954; Akabane, 1958; Harvey, 1964; Kundu, 1965; Castelli and Barron, 1969; Kakinuma et al., 1969; Castelli and Guidice, 1972). In some studies (see e.g. Akabane, 1956) no distinction was made between the impulsive and gradual rise and fall (GRF) bursts. This is unfortunate because the GRF bursts show a much larger center-to-limb variation in occurrence frequency than the impulsive bursts. For the impulsive bursts, Kakinuma et al. (1969) found 


\section{IMPULSIVE BURST AT $9.4 \mathrm{GHz}$}

( 16 DEC 1967)
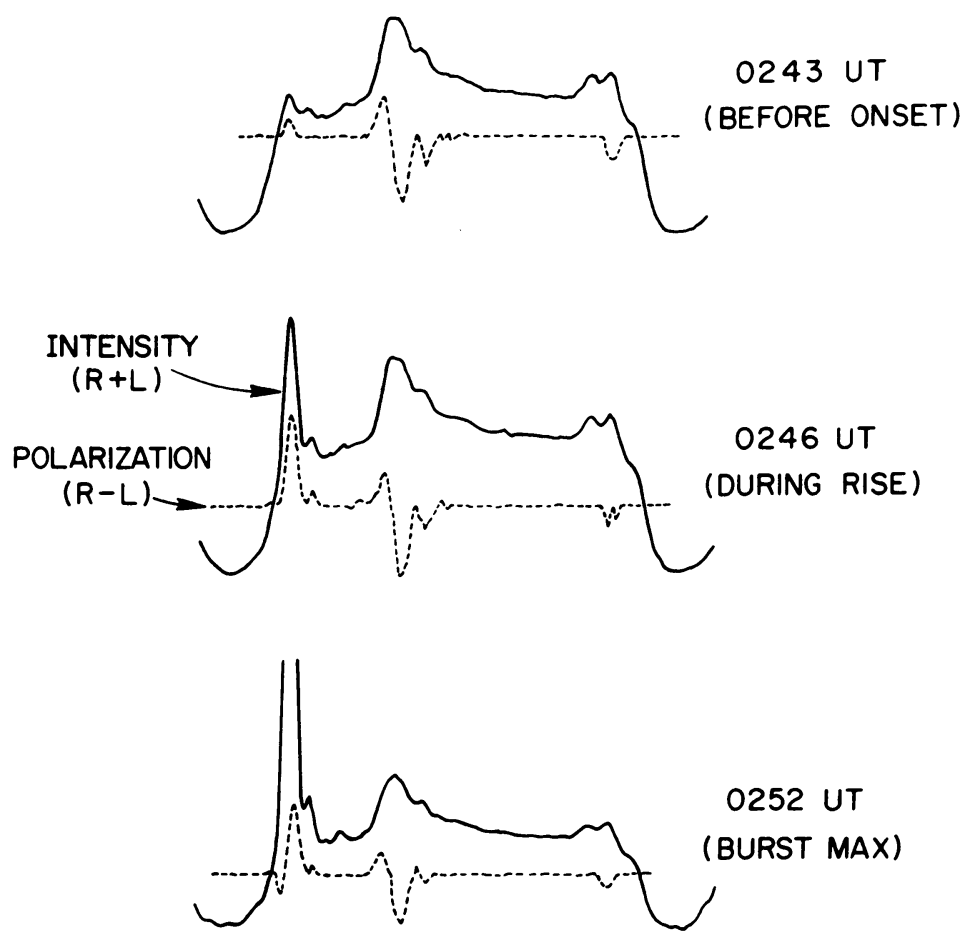

EAST JWEST

SOLAR DISC

Fig. 12. The time development of the intensity (solid line) and circular polarization (dashed line) at 9.4 $\mathrm{GHz}$ during the increasing phase of the impulsive burst on 16 December 1967, Initially the right hand plarization $(R)$ was dominant in the whole radio source (located near the east limb). However near the burst maximum the left handed polarization $(L)$ dominated in the eastern part of the source (Enome et al., 1969).

that, as compared to the bursts associated with flares located near the center of the solar disc, the intensity of bursts for flares located at a central meridian distance (CMD) of $\sim 75^{\circ}$ is about $0.56,0.58,0.65$ and 0.51 at $9.4,3.75,2.0$ and $1.0 \mathrm{GHz}$ respectively. This decrease in the observed emission for flares at large CMD could be due to the unisotropic emission by the source itself and/or due to the attenuation of the emission by an absorbing layer above the source. In the latter case, the observations indicate that the optical depth of the source at $\mathrm{CMD}=0$ is $\sim 0.2$, nearly independent of the emission frequency in $1.0-9.4 \mathrm{GHz}$ range. No significant center-tolimb variation in the degree of polarization of the impulsive microwave bursts has been observed (Kakinuma et al., 1969).

The recent high resolution measurements by Enome et al. (1969) show that the size of the microwave source is $\sim 0.5^{\prime}$. For the microwave burst associated with the hard 
X-ray burst (Figure 8) observed by Takakura et al. (1971), Tanaka and Enome (1971) found that at $3.75 \mathrm{GHz}$ the width of the radio source was $<1.8^{\prime}$. The observations for this event are consistent with the location and size of the hard X-ray and microwave sources being essentially the same.

The altitude of the microwave source is believed to be $\gtrsim 2 \times 10^{4} \mathrm{~km}$ above the photosphere (Kundu, 1965). This estimate is based on observations of behind-thelimb flares (Covington and Harvey, 1961a; Bruzek, 1964; Enome and Tanaka, 1971). The small optical depth of the source deduced from the center-to-limb variation of the microwave bursts is also consistent with a high altitude for the microwave source.

The correlation between the impulsive hard X-ray and microwave emissions was noticed earlier by Peterson and Winckler (1959) and Kundu (1961). This correlation has now been confirmed by observations of a large number of flare events (Arnoldy et al., 1967, 1968a, b; Kane and Anderson, 1970; Kane, 1972a; McKenzie, 1972). Although the correlation is most apparent for the times of microwave and X-ray maxima, quantitative relationship between the $\mathrm{X}$-ray and microwave fluxes also exists (Figure 13). The ratio of the peak X-ray $(\gtrsim 20 \mathrm{keV})$ and microwave $(\sim 3 \mathrm{~cm})$ energy fluxes is given by

$$
R_{x m}=\frac{\xi(\gtrsim 20 \mathrm{keV})}{\xi(\sim 3 \mathrm{~cm})} \sim 10^{2} .
$$

The type III radio bursts represent energetic electrons passing outward through the corona. Since the early work of Malville (1962) the flare associated type III bursts have been recognized as an impulsive phase phenomenon. The fact that electrons in essentially the same energy range (10-100 keV) are responsible for the impulsive hard $\mathrm{X}$-ray and type III radio emission led de Jager $(1960,1962)$ to predict a close relationship between these two emissions. Although correlated type III and hard X-ray bursts were occasionally observed (Anderson and Winckler, 1962; Winckler $e$ t al., 1961), in general no significant relationship was found (Kundu, 1961, 1965). The recent high time resolution $(\sim 2.3 \mathrm{~s})$ measurements of impulsive hard X-ray bursts indicate that even though the overall correlation between the hard X-ray and type III bursts is only $\sim 30 \%$, the relationship between these two emissions is probably more intimate than was believed in the past (Kane, 1972b). This is particularly true about the type III bursts which extend into the decimeter range. Figure 14 shows an example of a type III radio event which seems to be well correlated even in some of the fine structure with an impulsive X-ray burst observed with the OGO-5 satellite. In this particular case, there is a suggestion that individual groups of bursts in the type III emission have their counterparts in the intensity spikes in the associated $\mathrm{X}$-ray burst. This indicates that the true time constant for $\mathrm{X}$-ray intensity variation during the flash phase may in fact be $\sim 0.1 \mathrm{~s}$.

\section{Extreme Ultraviolet Observations}

The impulsive 10-1030 $\AA$ EUV emission during solar flares was first deduced in- 


\section{CORRELATION OF PEAK X-RAY AND RADIO FLUX}

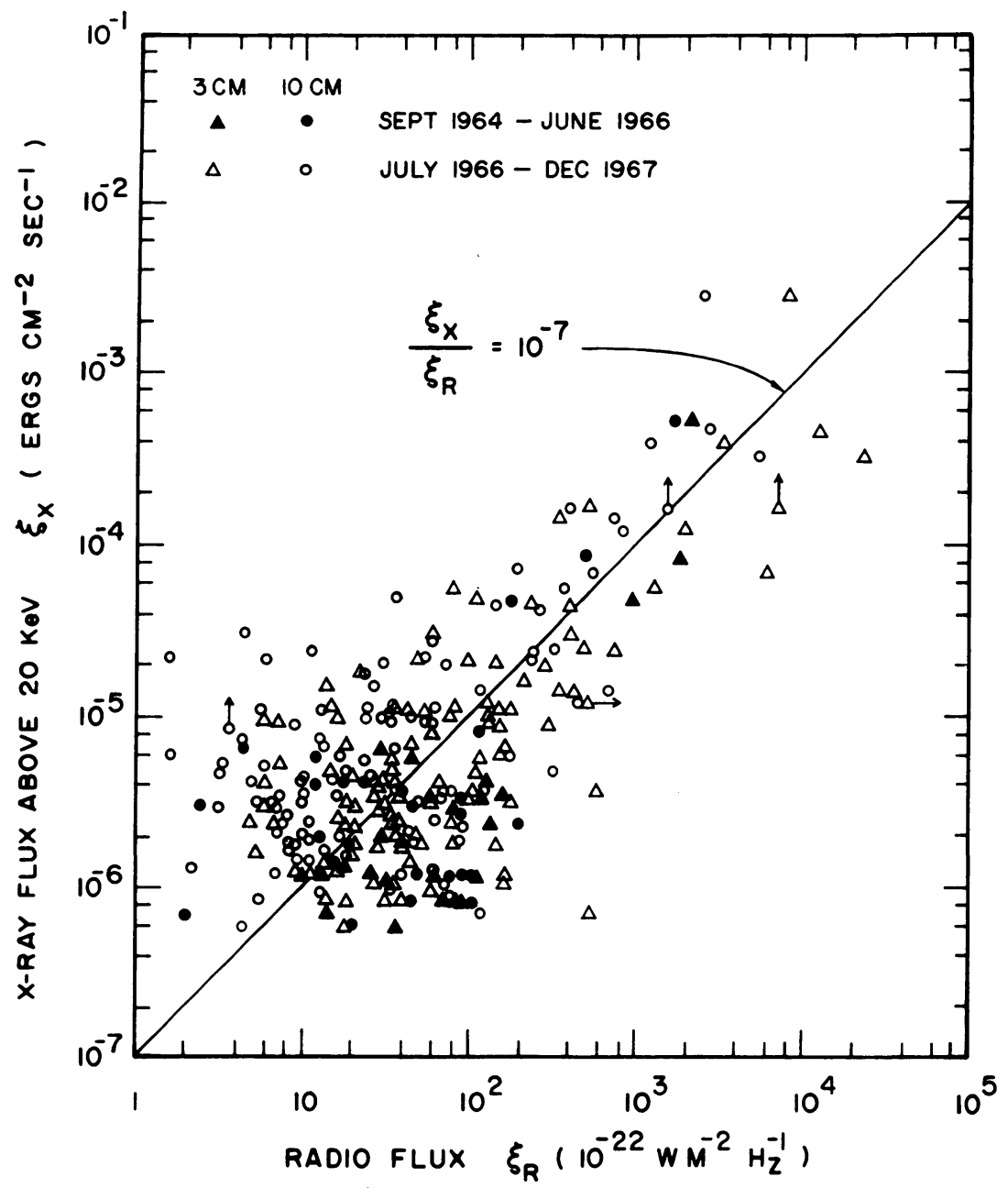

Fig. 13. A regression plot of the peak energy flux of X-rays $\gtrsim 20 \mathrm{keV}$ measured by the OGO-1 and OGO-3 ionization chambers and the peak energy flux of the microwave emission. Two separate time intervals are considered: September, 1964-June, 1966 (full symbols) and July, 1966-December, 1967 (open symbols). There is no observable difference between the regression lines for these two time intervals (Arnoldy et al., 1968a, b, c; Kane and Winckler, 1969a, b).

directly by Donnelly $(1968,1969,1971)$ from the observations of the ionospheric effect 'Sudden Frequency Deviation' (SFD) and a good correlation was found between the impulsive X-ray, EUV and microwave bursts (Kane and Donnelly, 1971). Since then satellite measurements of the line spectrum (Hall and Hinteregger, 1969; Castelli and Richards, 1971; Hall, 1971; Wood et al., 1972; Wood and Noyes, 1972; Donnelly et al., 1973) and the broad band emission (Kelly and Rense, 1973) have con- 


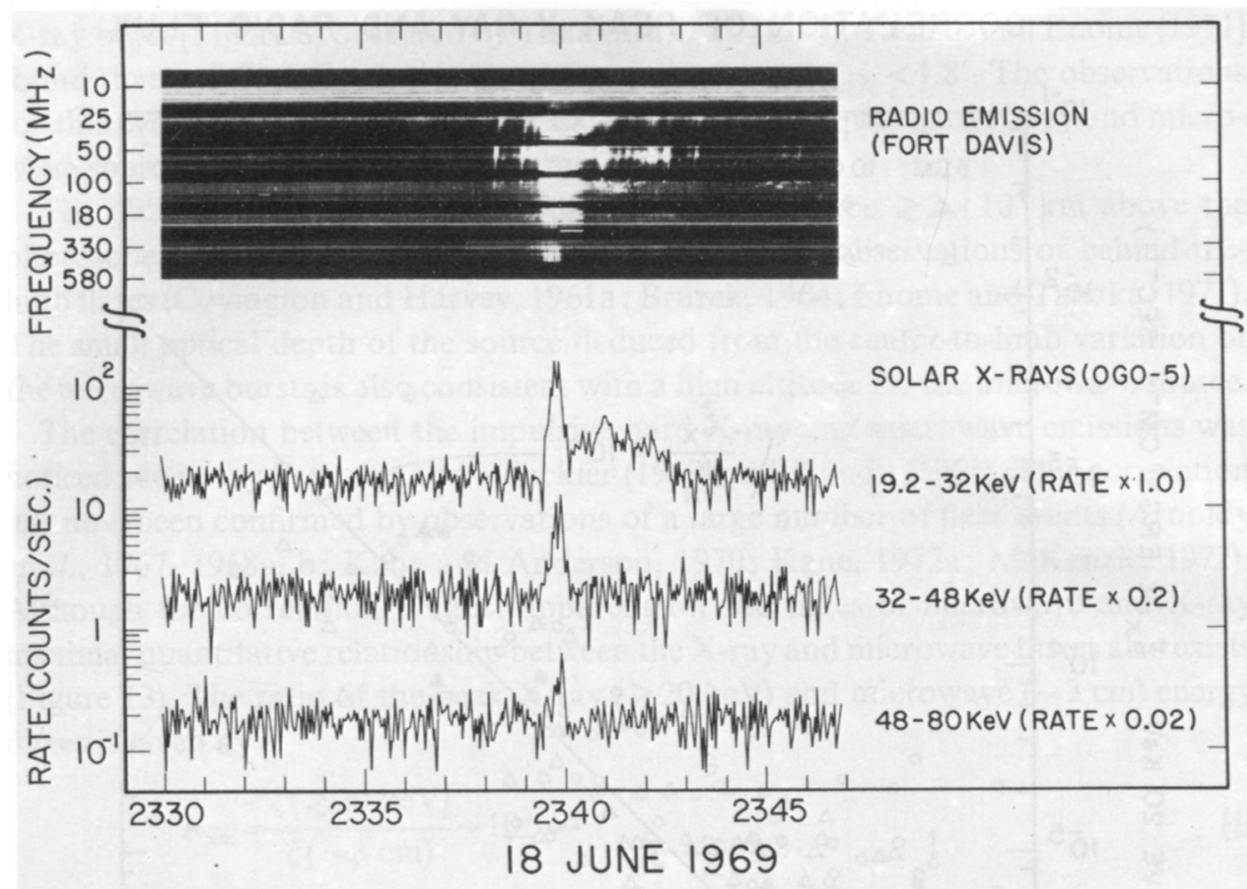

Fig. 14. An example of a type III radio event which seems to be well correlated even in some fine structure with an impulsive $X$-ray burst observed with the OGO-5 satellite. The individual groups of bursts in the type III emission seem to have their counterparts in the intensity spikes in the X-ray emission.

firmed the existence of the impulsive EUV emission by direct observations and have given some insight into the characteristics of energy transport in the flare region. The ground based and satellite observations of the 10-1500 $\AA$ flare emission have been recently reviewed by Donnelly (1973) and Noyes (1973) respectively. Here we will only briefly summarize the characteristics of the impulsive EUV bursts.

Figure 15 shows an impulsive burst at $630 \AA$ (O v) reported by Castelli and Richards (1971). Similar bursts have been observed in other EUV lines such as $304 \AA$ (He II $\mathrm{L} \alpha)$ and $972.5 \AA(\mathrm{H} \mathrm{L} \gamma)$ and broad band EUV emission. A comparison of the rise times of the EUV and X-ray emission is shown in Figure 16. There is an approximate proportionality between the peak EUV and X-ray energy fluxes. This is illustrated in Figure 17 (Kane and Donnelly, 1971).

Since all the desirable features, such as a high time and spatial resolution and observations of a large number of EUV lines and continuum over a wide spectral range, are not simultaneously available for many flare events, the available information is far from complete. Therefore only a very crude picture of the EUV flare can be constructed at this time. We present below what appears to be some general properties of the impulsive EUV emission:

(1) The EUV emission from flares consists of two components, viz. impulsive and gradual. (2) The increase in the impulsive component coincides in time with the in- 
crease in other impulsive emissions, such as hard X-ray and microwave emission. (3) The rise time of the broad band 10-1030 $\AA$ emission is comparable to that of $\sim 10 \mathrm{keV} \mathrm{X}$-rays. (4) For flares of $\mathrm{H} \alpha$-importance $\$ 3$, the peak energy flux in 10 $1030 \AA$ range varies from $0.1-10 \mathrm{ergs} \mathrm{cm}^{-2} \mathrm{~s}^{-1}$ at $1 \mathrm{AU}$. (5) The ratio of the peak EUV flux to the peak flux of X-rays $\gtrsim 10 \mathrm{keV}$, which is $\sim 10^{5}$ for flares located near

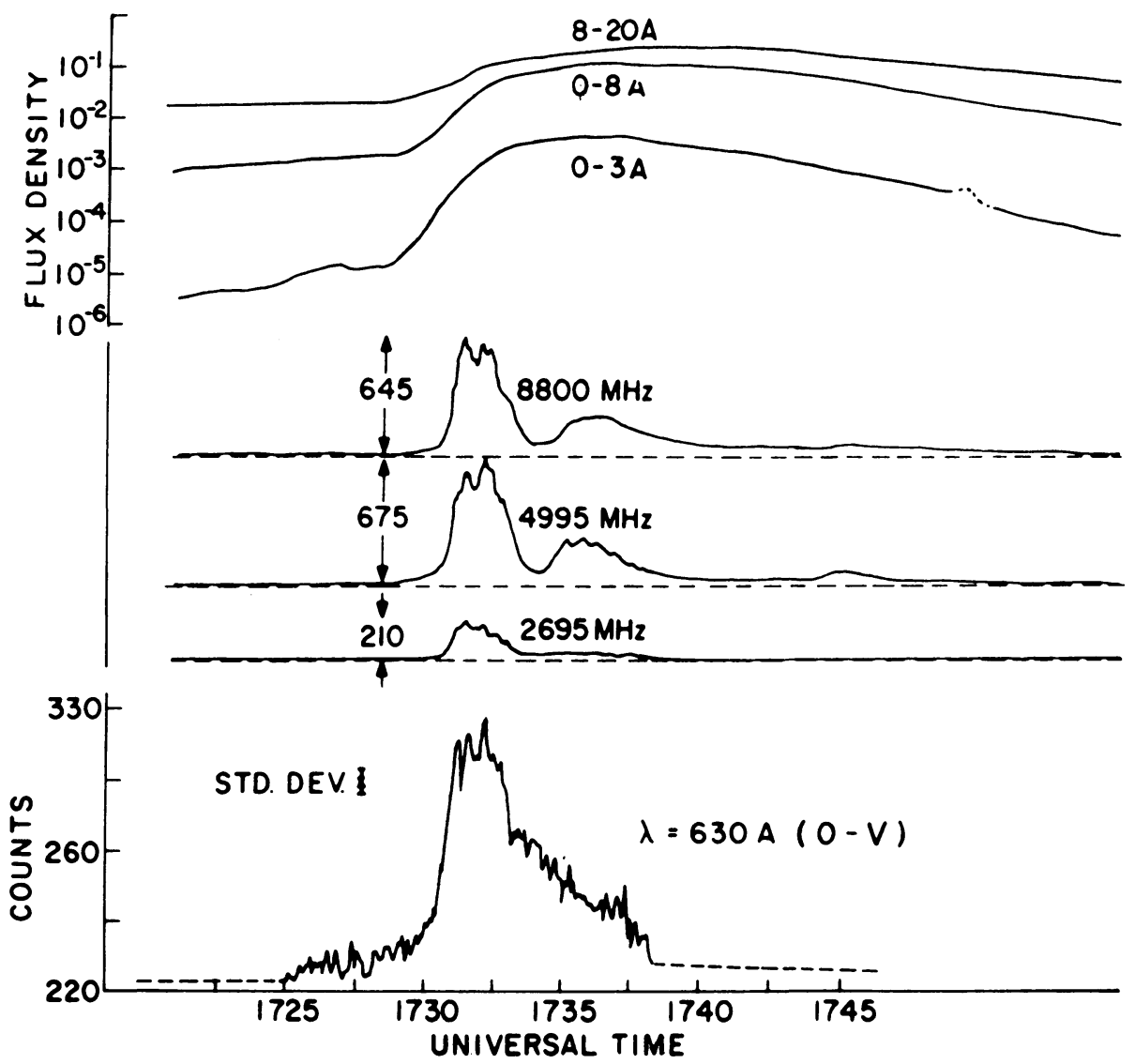

Fig. 15. An impulsive burst at $630 \AA(\mathrm{O} \mathrm{v})$ well correlated with the associated microwave emission (Castelli and Richards, 1971).

the central meridian, decreases with the increase in the central meridian distance of the flare. (6) The impulsive EUV source is located at an altitude $<10^{4} \mathrm{~km}$ above the photosphere. (7) The lines enhanced during the impulsive phase represent characteristic temperatures varying from $10^{4} \mathrm{~K}$ (chromospheric) to $1.5 \times 10^{6} \mathrm{~K}$ (coronal). (8) The relative enhancement of the various lines is approximately the same at the emission maximum although there is some evidence that the 'transition zone lines' tend to increase more than the coronal lines. (9) At a given time, the increase in the total line emission is of the same order of magnitude as the increase in the total continuum emission. 


\section{Optical Observations}

Since the discovery of solar flares, the optical observations have played a very important role in the classification and understanding of the flare phenomenon. Excellent reviews of solar flare observations made before 1963 have been given by Ellison (1963) and Smith and Smith (1963). The more recent optical observations of flares have been discussed by Švestka $(1969,1970)$ and Zirin $(1973)$. Since we are primarily

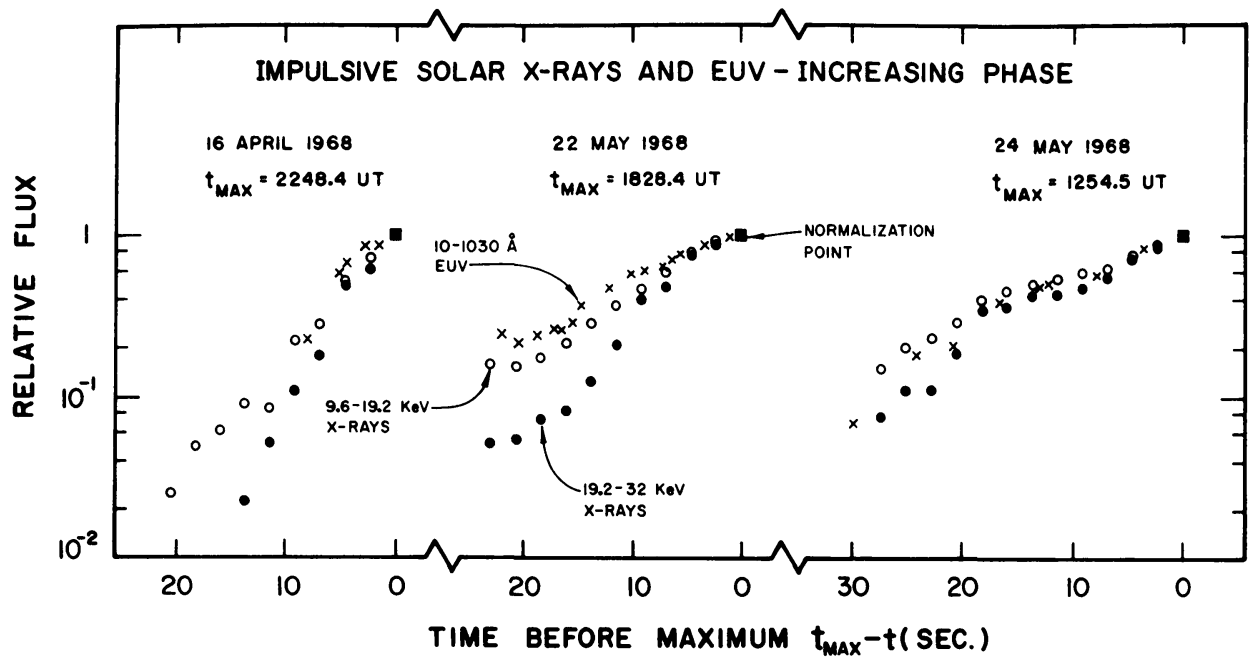

Fig. 16. A comparison of the rise times of the broad band EUV and hard X-ray emission for three impulsive bursts. The rise time of EUV emission is comparable to that of $\sim 10 \mathrm{keV} \mathrm{X}$-rays

(Kane and Connelly, 1971).

concerned here with the impulsive phase of flares, the observations most relevant to the present discussion are those made with a high time and spatial resolution.

\section{1. 'MONOCHROMATIC' OBSERVATIONS}

Most 'monochromatic' (narrow band) observations of the impulsive optical emission have been made in $\mathrm{H} \alpha$. Few observations of the $\mathrm{K}$ line of calcium have also been made. However the high resolution observations made at the Big Bear Observatory show that there is no significant difference in the appearance of flares in $\mathrm{H} \alpha$ and the $\mathrm{K}$ line (Zirin, 1973). Therefore only the $\mathrm{H} \alpha$ observations will be discussed here.

In the past, the observations of the impulsive $\mathrm{H} \alpha$ emission from flares have been discussed in terms of two phases, viz. the flash phase and the explosive phase. Following Ellison $(1949,1963)$ the flash phase is usually considered as the time of rapid increase in $\mathrm{H} \alpha$ line-width associated with the rapid increase in the $\mathrm{H} \alpha$ emission during the early (pre-maximum) part of a flare. On the other hand, the explosive phase is marked by an abrupt increase in the $\mathrm{H} \alpha$ flux caused primarily by the rapid expansion of the flare borders (Moreton, 1964). A common factor in the two phases is the rapid increase in the observed $\mathrm{H} \alpha$ flux. Whereas there is no specific requirement in the flash 
phase on the cause of the increase in $\mathrm{H} \alpha$ flux, the explosive phase requires that this increase be primarily due to the expansion of the flare borders.

The flash phase of small flares and their correlation with the type III radio bursts has been studied by Malville (1962). Following the early work of Covington and Harvey (1961b), the explosive phase and its relationship with the impulsive X-ray, EUV and microwave emission has been studied by Moreton (1964), Davies and Donnelly (1966), Angle (1968), and Harvey (1971). The observed good correlation clearly

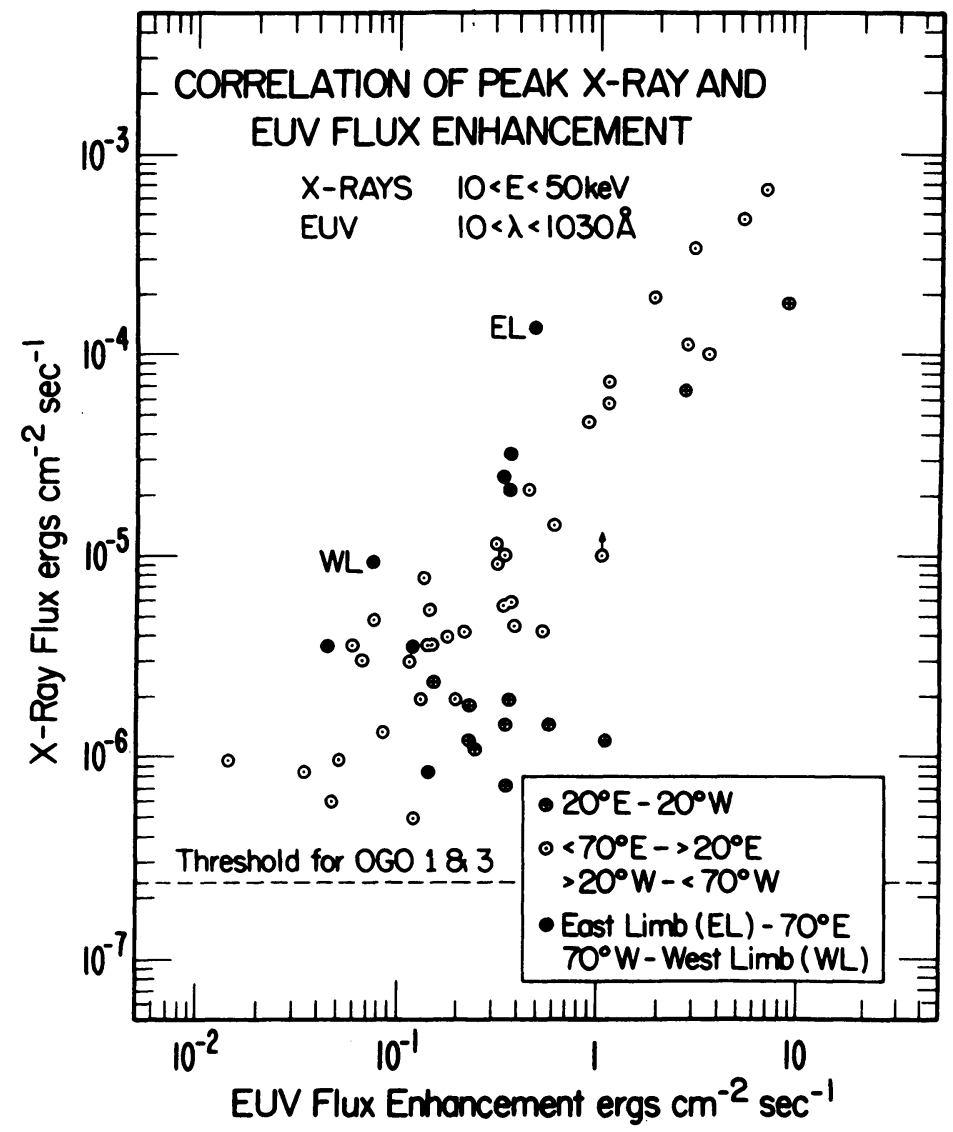

Fig. 17. A regression plot of $10-1030 \AA$ EUV flux and $10-50 \mathrm{keV} \mathrm{X-ray} \mathrm{flux} \mathrm{observed} \mathrm{at} \mathrm{the} \mathrm{maxima} \mathrm{of}$ impulsive flare events (Kane and Donnelly, 1971).

indicates that the explosive phase is nearly coincident with the impulsive phase defined here.

It has been known for some time that the impulsive phase may occur only in a small part of an $\mathrm{H} \alpha$-flare (Dodson et al., 1956; Dodson and Hedeman, 1968; Harvey, 1971; Vorpahl, 1972). An example of the development of a large flare $(\mathrm{H} \alpha-$ importance $\gtrsim 3$ ) is shown in Figure 18 (Harvey, 1971). Among the A, B and E sections of the flare only the section $E$ of the flare had an explosive phase. A similar effect 


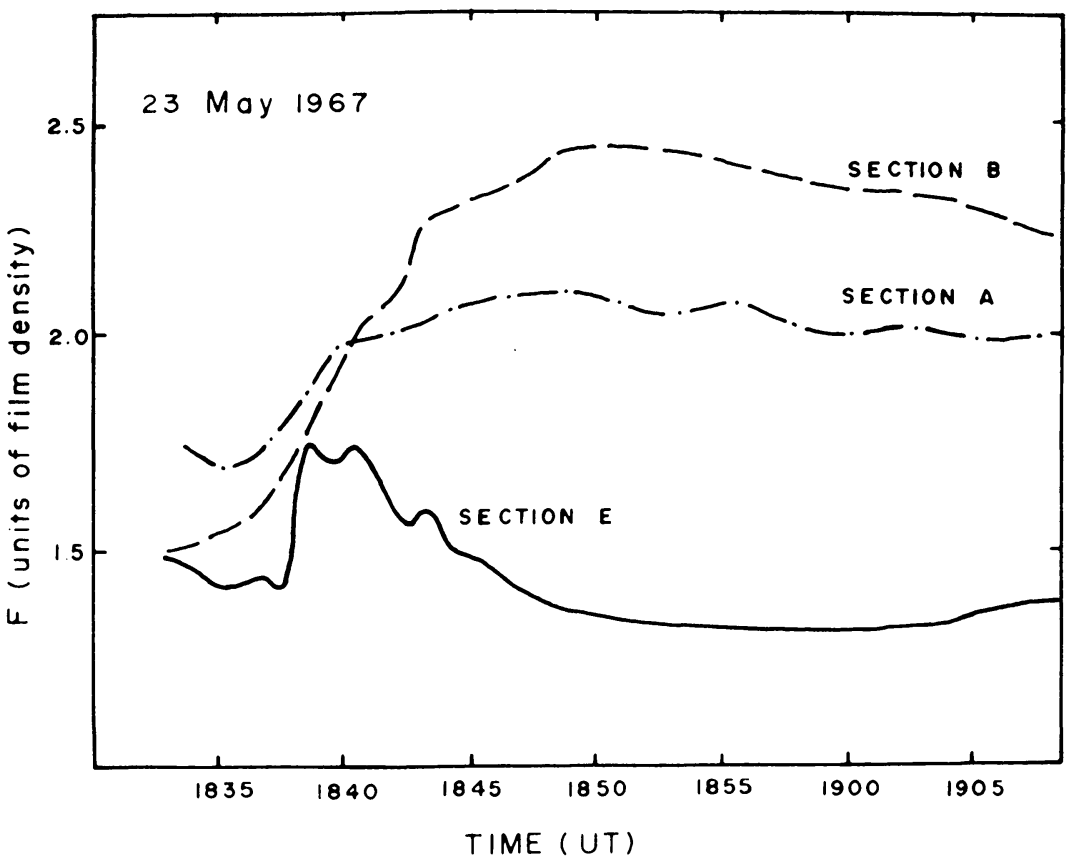

Fig. 18. The development of different regions (A, B, E,) of a large $\mathrm{H} \alpha$-flare with time. Only the region $\mathrm{E}$ had an explosive phase (Harvey, 1971).

can be seen in Figure 19 where the development of a relatively small flare is illustrated (Vorpahl, 1972).

The impulsive phase of more than 55 flare events, most of which were of $\mathrm{H} \alpha$ importance $\lesssim 1$, has been recently studied by Zirin et al. (1971), Vorpahl (1972), and Zirin (1973). Their principal findings may be summarized as follows: (1) The $\mathrm{H} \alpha$ kernels which undergo impulsive development are much more intense than the remaining part of the flare. (2) The $\mathrm{H} \alpha$ intensity of the impulsive kernels begins to increase 20-30 s before the onset of the impulsive X-ray increase and peaks approximately at the time of the X-ray maximum. (3) The increase in intensity of the impulsive kernels is associated with an increase in the $\mathrm{H} \alpha$ line-width. However, this increase in line-width is confined to the $\mathrm{H} \alpha$ emission from the impulsive kernels only; the remaining flare area is relatively unaffected. (4) The total energy radiated in $\mathrm{H} \alpha$ by an impulsive kernel is $\sim 5 \times 10^{25}$ erg in a small flare. (5) The impulsive kernels are located lower in the chromosphere $(\$ 5000 \mathrm{~km}$ altitude) than the remaining part of the $\mathrm{H} \alpha$ flare. (6) Although outward motion is sometimes seen simultaneously with, or following, the impulsive phase, an impulsive burst is more likely to occur when the optical flare exhibits little expansion of material or light. (7) The effective diameter of the $\mathrm{H} \alpha$ kernel is $3000-6000 \mathrm{~km}$ and normally represents $<\frac{1}{8}$ to $\frac{1}{2}$ of the total flare area at the $\mathrm{H} \alpha$ maximum. (8) The occurrence of the impulsive phase has little to do with the size of the $\mathrm{H} \alpha$-flare. (9) Bright kernels invariably occur near steep magnetic field 
gradients. (10) Almost all flares are bipolar. When only one kernel is present, the brightness occurs only on one side of the neutral line. Multiple kernels, when present, are located in areas of opposite polarity and are apparently connected by the same field lines.

\subsection{BROAD-BAND OBSERVATIONS}

The most common broad band observations are the observations of 'white light flares' (DeMastus and Stover, 1967), which are usually very large and complex flares. For small and medium flares with which we are primarily concerned here, the only

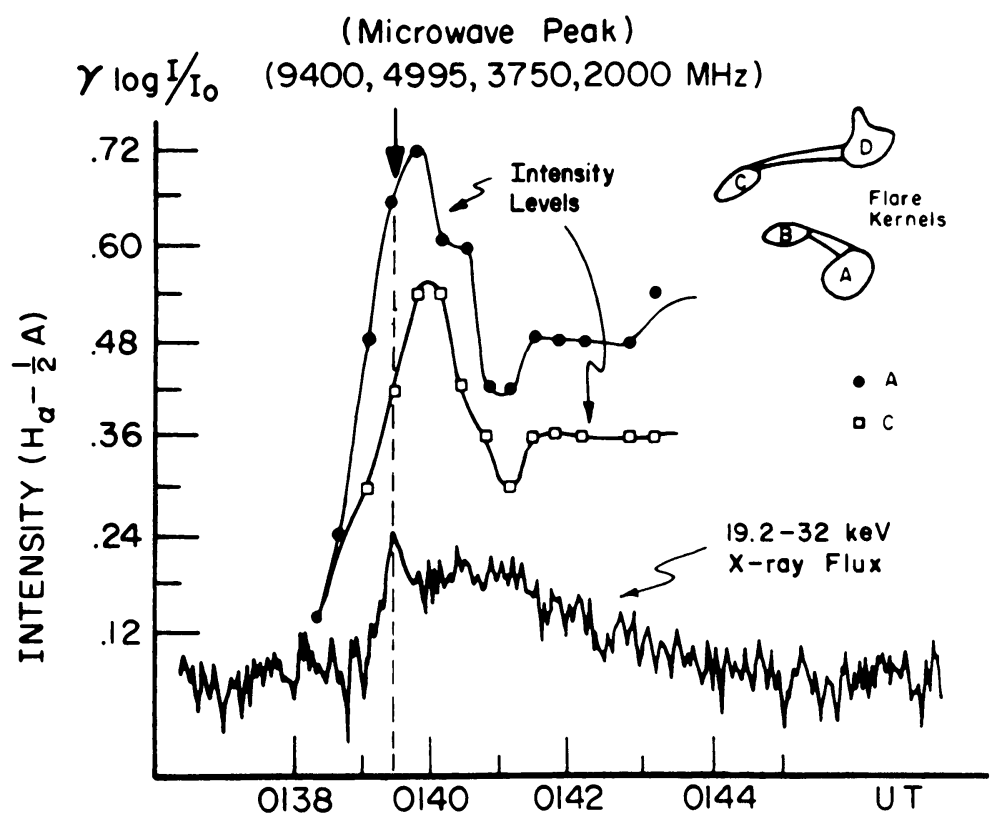

Fig. 19. The development of different regions or kernels (A, B, C, D) of a small $\mathrm{H} \alpha$-flare with time. Only the kernels $A$ and $C$ had an impulsive phase (Vorpahl, 1972).

broad band measurements reported in the literature are those made by Zirin (1972) in the $15 \AA$ band centered at $3835 \AA$. They did not find any increase in the emission of $3835 \AA$ even in fairly large flares indicating that in most flares there is no significant heating of the lower chromosphere at a height where the density is $\sim 10^{17}$ particles $\mathrm{cm}^{-3}$. However, in the very large flares of 2 August 1972, Tanaka and Zirin (1973) did find a large increase in the $3835 \AA$ emission in good time coincidence with the hard X-ray and microwave enhancements.

\section{Summary and Discussion}

Table II summarizes the characteristics of the various electromagnetic emissions during the impulsive phase. The quantities such as the rise time and peak energy flux 
are based on observations discussed above. Some characteristics of the source region, such as altitude above the photosphere, linear dimension and double-source nature are also based primarily on observations. In some cases very few observations of a given quantity are available. For example, the altitude of the hard X-ray source has been observed only in one well documented behind-the-limb flare. However, in view of the studies involving comparison of the impulsive X-ray and interplanetary electron measurements (Datlowe and Lin, 1973) and the fact that no known observation is inconsistent with an altitude of $\sim 10^{4} \mathrm{~km}$ for the hard X-ray source, we have included this altitude in Table II as the best estimate presently available. The quantities such as the ion density $n_{i}$, neutral density $n_{0}$ and Temperature $T$ for the radiation sources are deduced on the basis of the observed spectral and temporal characteristics of a given radiation and a plausible theoretical model specifically applicable to

TABLE II

Impulsive phase electromagnetic emissions

\begin{tabular}{|c|c|c|c|c|c|}
\hline \multirow[t]{2}{*}{ Characteristic } & \multicolumn{5}{|l|}{ Radiation } \\
\hline & Microwave & $\begin{array}{l}\text { X-rays } \\
(\gtrsim 20 \mathrm{keV})\end{array}$ & $\begin{array}{l}\text { Type III } \\
\text { decimeter } \\
\text { and meter }\end{array}$ & EUV & $\mathbf{H} \alpha$ \\
\hline Emission process & Synchrotron & $\begin{array}{l}\text { Brems- } \\
\text { strahlung }\end{array}$ & $\begin{array}{l}\text { Plasma } \\
\text { hypothesis }\end{array}$ & $\begin{array}{l}\text { Bound-bound } \\
\text { free-bound } \\
\text { and free-free }\end{array}$ & Bound-bound \\
\hline Rise time (s) & $\sim 1$ & $\$ 1$ & $\sim 0.1$ & $\sim 10$ & $\sim 10$ \\
\hline $\begin{array}{l}\text { Peak energy flux relative to } \\
\text { peak X-ray energy flux } \\
\text { Total energy radiated in a }\end{array}$ & $\sim 10^{-2}$ & 1 & $\sim 10^{-4}$ & $\sim 10^{5}$ & $\sim 10^{2}$ \\
\hline $\begin{array}{l}\text { small flare (ergs) } \\
\text { Source region: }\end{array}$ & $\sim 10^{21}$ & $\sim 10^{23}$ & $\gtrsim 5 \times 10^{18}$ & $\sim 10^{28}$ & $\sim 5 \times 10^{25}$ \\
\hline Altitude $h(\mathbf{k m})$ & $\sim 10^{4}$ & $\sim 10^{4 \mathrm{a}}$ & $\gtrsim 5 \times 10^{4}$ & $\lesssim 5 \times 10^{3}$ & $\sim 5 \times 10^{3}$ \\
\hline Linear dimension $L(\mathrm{~km})$ & $\sim 10^{4}$ & $?$ & $?$ & ? & $\sim 5 \times 10^{3}$ \\
\hline Double-source nature & Yes & $?$ & - & $?$ & Yes \\
\hline Ion density $n_{i}\left(\mathrm{~cm}^{-3}\right)$ & $\sim 10^{10}$ & $\sim 10^{10 \mathrm{a}}$ & $\lessgtr 10^{9}$ & $\gtrsim 10^{11}$ & $\gtrsim 10^{11}$ \\
\hline Neutral density $n_{0}\left(\mathrm{~cm}^{-3}\right)$ & $\sim 0$ & $\sim 0^{\mathrm{a}}$ & $\sim 0$ & $\gtrsim 10^{12}$ & $\gtrsim 10^{12}$ \\
\hline Temperature $T(\mathrm{~K})$ & $\sim 10^{6}$ & $\sim 10^{6 \mathrm{a}}$ & $\sim 10^{6}$ & $\$ 10^{4}$ & $\$ 10^{4}$ \\
\hline
\end{tabular}

- Although there is considerable uncertainty in these numbers, they are the best estimates available at the present time.

that radiation source. For example the value of $n_{i} \sim 10^{10} \mathrm{~cm}^{-3}$ for the microwave source is based on the theoretical models of Takakura $(1968,1973)$, Holt and Ramaty (1969), and Ramaty and Petrosian (1972) which are consistent with the observations.

A number of important features of the impulsive phase may be noted from Table II. Most of the radiated energy is emitted in the EUV range. The energy radiated as optical emission is not well known at the present time. The $\mathrm{H} \alpha$ emission carries a relatively small fraction of the energy. The study of white light flares recently made by McIntosh and Donnelly (1972) indicates that the energy radiated as white light 
(3500-6500 $\AA$ ) is probably of the same order of magnitude as the energy in the broad band EUV emission.

Although the energy radiated as microwave, $\mathrm{X}$-ray and type III radio emission is relatively small, these emissions are the evidence for the existence of non-thermal electrons in the flare region. 'Thermal' electron spectra have been invoked from time to time to explain fully or in part the observed hard X-ray spectra (Chubb et al., 1966; Chubb, 1972; Brown, 1973). It was also reported that the emission measure remains constant during an X-ray burst (Hudson et al., 1969). This was, however, found to be an instrumental effect (Kane and Hudson, 1970). The power law X-ray spectrum, its rapid time variations and the close relationship with the microwave and type III radio emissions strongly suggest that the electron spectrum inside the hard X-ray source is non-thermal. The X-ray polarization measurements (Tindo et al., 1970, 1972a, b) are also consistent with this interpretation.

The rapid time variations in the hard X-ray, microwave and type III radio emission indicate an electron acceleration process which is continuous in time or more probably a continuous series of impulses with a time constant $\$ 1 \mathrm{~s}$. Although the parameters of the electron acceleration spectrum deduced from the hard X-ray observations depend on the details of the assumed X-ray source model, some characteristics of the acceleration process can still be determined. Table III shows the properties of the electron acceleration process deduced from the X-ray observations summarized in Table $I$. The efficiency of acceleration is expressed as the ratio of the total kinetic energy of the accelerated electrons $\gtrsim 20 \mathrm{keV}$ to the total energy (thermal and non-thermal) of the flare. In constructing Table III a thin-target model for the hard X-ray source with continuous electron injection has been used (Kane and Lin, 1972; Kane, 1973a, b). If, instead, a thick-target model is assumed (Hudson, 1972a, b; Syrovatskii and Shmeleva, 1971), the deduced electron spectrum exponent $\varphi$ will be larger by $\sim 1$, but the overall characteristics of the acceleration process indicated in Table III will remain relatively unchanged. Two important facts are revealed by Table III : (1) the acceleration process has a very short time constant, (2) the total kinetic energy of the accelerated electrons $\gtrsim 20 \mathrm{keV}$ is comparable to the total energy radiated during the impulsive phase. Thus the observations are consistent with a model of the impulsive

TABLE III

Acceleration of electrons in small solar flares

Nature of the process

Characteristic time

Total duration

Electron spectrum (averaged over $\sim 2$ s)

Limits on exponent $\varphi$

Time variation of $\varphi$

Probability of occurrence of a given $\varphi_{0}$ in a flare

Total kinetic energy of electrons $\gtrsim 20 \mathrm{keV}$

Efficiency of acceleration

Probable location of the acceleration region

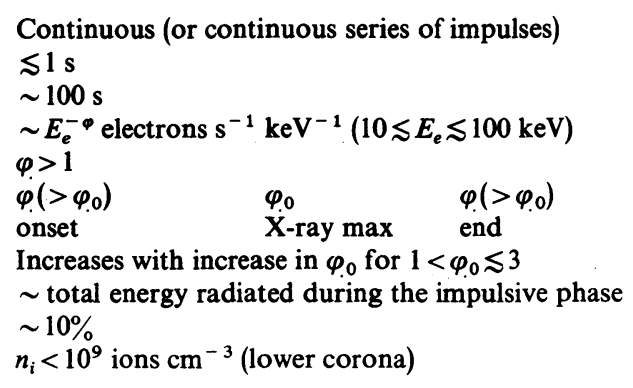


phase in which energetic electrons play a central role and provide most of the energy required for the various electromagnetic emissions during the impulsive phase.

At present there seems to be no clear evidence for the unisotropic emission of either the hard X-ray or the microwave emission. Models of hard X-ray source with highly directional beams of energetic electrons, similar to those suggested earlier by de Jager and Kundu (1963), predict a relatively high degree of energy dependent polarization and unisotropy in the hard X-ray emission (Korchak, 1967; Elwert and Haug, 1970; Petrosian, 1973; Phillips, 1973).,Polarization measurements reported by Tindo et al. (1972a, b) are consistent with beams of electrons moving radially on the Sun. However, the absence of significant center-to-limb variation in the observed location of the $\mathrm{X}$-ray producing $\mathrm{H} \alpha$-flares indicates that the unisotropy in the X-ray emission, if present, is relatively small. Hard X-ray observations with two or more spacecraft separated widely in heliographic longitude will be required before this question can be resolved satisfactorily.

The microwave as well as $\mathrm{H} \alpha$ emission shows a double-source structure. Thus far it has not been possible to resolve a similar structure in the EUV and X-ray sources. The double-source structure is sometimes considered as evidence for a magnetic field structure where the two radiation sources are connected by closed field lines (Vorpahl, 1972). However, it is also possible that the two sources are located on open field lines which pass through the region of primary energy release. Thus, the observations do not necessarily require an acceleration region located in the closed field region.

The observations are in general agreement with models of solar flares, such as those proposed by Alfvén and Carlqvist (1967), Sturrock (1968, 1973), Syrovatskii (1969), and Takakura (1971), in which induced or quasi-static electric fields accelerate particles during the impulsive phase. Except for a model recently discussed by Smith (1973), most flare models do not predict the spectrum of the accelerated particles and hence direct comparison with the observations is not possible. A similar difficulty arises with respect to the basic time constant for the acceleration process. The observations require that the basic time constant for acceleration be $\lesssim 1 \mathrm{~s}$ and the rate of energy release in a small flare be $\sim 10^{27} \mathrm{erg} \mathrm{s}^{-1}$.

\section{Models of the Flash Phase}

Although the role of energetic $(>1 \mathrm{MeV})$ protons in the impulsive phase emissions has been emphasized from time to time (Švestka, 1971; Najita and Orall, 1970; Biswas and Radhakrishnan, 1973), the principal characteristic of the impulsive phase seems to be the acceleration of electrons to energies up to a few hundred $\mathrm{keV}$. This was earlier recognized by de Jager $(1960,1962)$ and Wild et al. (1963) and is still fully consistent with the observations described here. As suggested by Wild et al. (1963) and de Jager (1969), the acceleration of protons to energies greater than a few hundred $\mathrm{keV}$ probably takes place during a second phase of acceleration which occurs soon after the impulsive phase. New evidence to support such a two step acceleration process has been recently reported by Frost and Dennis (1971). We will therefore 
consider here electrons from a few $\mathrm{keV}$ to a few hundreds of $\mathrm{keV}$ as the principal component of the particles accelerated during the impulsive phase, keeping in mind that some protons in a similar energy range may also be accelerated.

There is a growing opinion that the accelerated electrons probably play a central role in the development of the impulsive phase (Anderson et al., 1970; Kahler et al., 1970; Lin and Hudson, 1971; Syrovatskii and Shmeleva, 1971; Zirin et al., 1971; Hudson, 1972a, b; Brown, 1971, 1973; Kane, 1973a, b; Lin, 1970, 1973, 1974a, b; Tanaka and Zirin, 1973). This inference is primarily based on the interpretation of the hard X-ray measurements.

The impulsive $\mathrm{X}$-ray emission is generally believed to be bremsstrahlung from energetic electrons interacting with the ambient solar gas. If the effect of Compton back scattering is neglected, it is relatively simple to deduce the instantaneous electron spectrum inside the $\mathrm{X}$-ray source from the observed $\mathrm{X}$-ray spectrum. The procedure has been described by Kane and Anderson (1970), Brown (1971) and others. In such a deduction the only unknown parameter is the ion density $n_{i}$ in the $\mathrm{X}$-ray source. However, the problem gets complicated and highly model dependent when we try to deduce the electron spectrum produced at the acceleration region. Several important assumptions are involved:

(1) Location of the acceleration region

(2) Structure of the magnetic field connecting the acceleration region to the hard $\mathrm{X}$-ray source

(3) Initial pitch angle distribution of the accelerated electrons

(4) Scattering of the electrons during their passage from the acceleration region to the X-ray source

(5) Average ion density $n_{i}$ in the X-ray source

(6) Energy loss processes for the electrons

Since most of these quantities are not known observationally, one is forced to take a 'black box' approach, i.e., substitute quantities such as deflection time and scale height for the detailed physical processes.

The models of the impulsive phase proposed so far can be classified into three types: Their basic properties are presented in Figure 20 and Table IV. The three models have many common features. For example, the emission processes are basically the same in all the three types of models. The hard X-ray and microwave emissions are produced as bremsstrahlung and synchrotron radiation respectively from the energetic electrons accelerated during the flash phase. The EUV and optical radiations are produced by bound-bound, free-bound and free-free emission processes. In all models the electron acceleration region is located in the lower corona and the EUV and optical emission sources are located in the lower chromosphere. The three models differ primarily in the details of the electron and/or energy transport from the acceleration region to the various emission sources.

In Model 1, which is similar to the models proposed by Hudson (1972a, b) and Syrovatskii and Shmeleva (1971), most of the accelerated electrons moving initially downward reach the lower chromosphere, deposit all of their energy there and 
produce localized heating. The hard-X-rays are produced by thick-target bremsstrahlung in the lower chromosphere. The location of the microwave source has not been specified. The motion of the electrons from the acceleration region towards the hard X-ray - EUV source may be in the form of a well directed beam or as a diffusion process depending on the magnetic field structure in the region and the amount of

CORONA

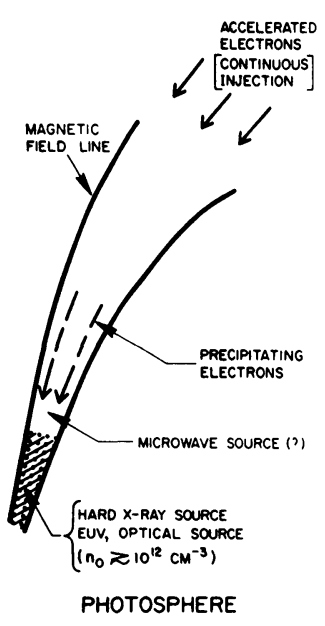

MODEL - 1
CORONA

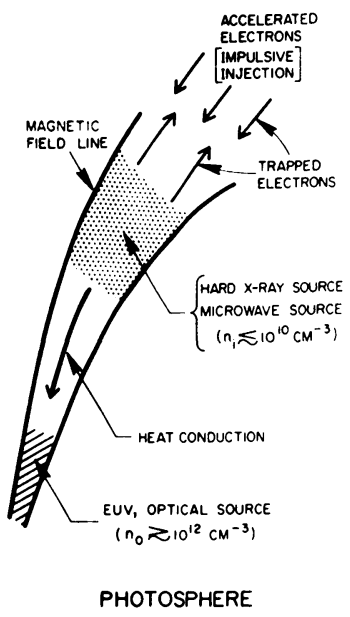

MODEL-2
CORONA

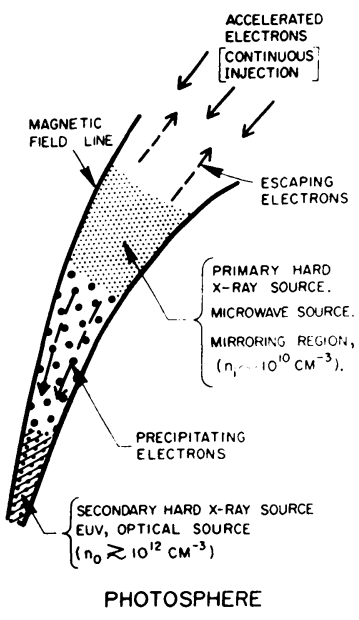

MODEL -3

Fig. 20. Models of impulsive (flash) phase in which electrons play a central role. Model 1 is similar to the models proposed by Syrovatskii and Shmeleva (1971) and Hudson (1972). Model 2 is similar to the model proposed by Takakura (1973). Model 3 has been proposed by Kane (1973b). In Model 3 the relative strength of the primary and secondary hard $\mathrm{X}$-ray sources may vary from event to event.

scattering the electrons have to undergo. In models where electron beams are considered, an implicit assumption is that the pitch angles $\alpha_{0}$ of the electrons leaving the acceleration region are relatively small and that the electrons do not undergo significant scattering. If $B_{0}$ is the magnetic field strength near the acceleration region and $B_{x}$ the field strength in the hard X-ray - EUV source, the required pitch angles are given by $\alpha_{0} \lesssim \sin ^{-1}\left(B_{0} / B_{x}\right)^{1 / 2}$. Electrons with larger pitch angles will mirrore before they reach the hard X-ray - EUV source. Inside the EUV source we expect $B_{x} \sim 10^{3} \mathrm{G}$. If the acceleration region is located in the lower corona where $B_{0} \sim 10 \mathrm{G}$, we find $\alpha_{0}<6^{\circ}$. Since some scattering is almost certain to occur, which will further reduce the required values of $\alpha_{0}$, we find that very small initial pitch angles for the accelerated electrons are required if the electrons are to move as a well directed beam.

At the other extreme is Model 2, which is similar to the one proposed by Takakura (1973). Here most of the accelerated electrons have large initial pitch angles and are trapped in a magnetic arch at a level where the ion density $n_{i}$ is $\$ 10^{10} \mathrm{~cm}^{-3}$. This is also the location of the hard $\mathrm{X}$-ray and microwave sources. The hard X-ray emis- 
TABLE IV

Models of flash phase in which electrons play a prominent role

\begin{tabular}{|c|c|c|c|}
\hline Characteristic feature & Model 1 & Model 2 & Model 3 \\
\hline $\begin{array}{l}\text { Location of acceleration region } \\
\text { Time dependence of }\end{array}$ & Lower corona & Lower corona & Lower corona \\
\hline acceleration process & Continuous & Impulsive & Continuous \\
\hline Hard X-rays (bremsstrahlung) & Thick-target & Thin-target & $\begin{array}{l}\text { Thin and thick-target } \\
\text { Thin-target dominant }\end{array}$ \\
\hline $\begin{array}{l}\text { Electron escape into corona } \\
\text { from hard X-ray source } \\
\text { Hydrogen (atom/ion) density } \\
\text { in emission sources }\left(\mathrm{cm}^{-3}\right)\end{array}$ & Negligible & Negligible & Substantial \\
\hline Microwave & $?$ & $10^{9}-10^{10}$ & $\sim 10^{10}$ \\
\hline Hard X-ray & $\gtrsim 10^{12}$ & $\sim 10^{10}$ & $\begin{array}{l}\text { Extended source } \\
\sim 10^{10} \text { (major part) } \\
\sim 10^{12} \text { (minor part) }\end{array}$ \\
\hline $\begin{array}{l}\text { EUV and optical } \\
\text { Transport from acceleration } \\
\text { region to EUV and optical } \\
\text { sources }\end{array}$ & $\gtrsim 10^{12}$ & $\gtrsim 10^{12}$ & $\gtrsim 10^{12}$ \\
\hline $\begin{array}{l}\text { Electron propagation } \\
\text { Energy transport }\end{array}$ & Complete & Negligible & Partial \\
\hline (primary mode) & Electrons $\gtrsim 5 \mathrm{keV}$ & Heat conduction & Electrons $\lesssim 10 \mathrm{keV}$ \\
\hline Crucial test for the model & $\begin{array}{l}\text { Low altitude of } \\
\text { hard X-ray source }\end{array}$ & $\begin{array}{l}\text { Short time } \\
\text { constant }(<10 \mathrm{~s}) \\
\text { for heat conduction }\end{array}$ & $\begin{array}{l}\text { Presence of escaped } \\
\text { electrons in lower } \\
\text { corona }\end{array}$ \\
\hline Reference & $\begin{array}{l}\text { Hudson (1972) } \\
\text { Syrovatskii and } \\
\text { Shmeleva (1972) }\end{array}$ & Takakura (1973) & Kane (1973b) \\
\hline
\end{tabular}

sion is produced by thin-target bremstrahlung. The energy for the EUV and optical emissions is transported from the acceleration region to the lower chromosphere by means of heat conduction.

Model 3, proposed by Kane (1973b) takes into account two possibilities:

(a) The electrons can mirror in the magnetic field before they reach the chromosphere and then escape into lower corona.

(b) Some of the mirroring electrons, particularly the lower energy ones ( $\$ 10 \mathrm{keV})$, can scatter into the loss cone and precipitate into the lower chromosphere.

In this model, the hard X-ray source extends from the lower chromosphere to the lower corona. The most intense part of the hard X-ray source is nearly coincident with the microwave source and has an ion density $n_{i} \sim 10^{10} \mathrm{~cm}^{-3}$. The X-ray emission in this part of the source is thin-target bremsstrahlung. The energy transport from the acceleration region to the lower chromosphere is through electrons with energy $\$ 10 \mathrm{keV}$. The value of $n_{i}$ inside the mirroring region may vary from one flare to another. When $n_{i}$ is $\ll 10^{10} \mathrm{~cm}^{-3}$, the hard X-ray, microwave, EUV and optical emission may not be detectable although the type III radio burst produced by the electrons escaping through the corona may be easily observed. This will correspond 
to the observations of type III bursts without optical flares (Malville, 1962; Teske et al., 1971; Axisa et al., 1973).

The most attractive feature of Models 1 and 2 is their relative simplicity. However, there are several difficulties with both these models. Model 1 predicts very low altitude of the hard X-ray (and microwave?) sources, which is not consistent with the available behind-the-limb flare observations. Another difficulty is the dominant thick-target $\mathrm{X}$-ray emission which is not favored by some observations involving hard X-rays and interplanetary solar electrons (Datlowe and Lin, 1973). In addition, Model 1 does not offer a natural explanation of the observations of type III bursts without impulsive hard X-ray, microwave, EUV and optical emission. Model 2 overcomes some of these difficulties. However, in order to explain the observed energy dependence of the decay of X-ray bursts, it requires a specialized ion density structure and pitch angle distribution of the electrons inside the X-ray source such as the one considered by Brown (1972). Moreover, it does not explain the similarity in the time variation of $\sim 10 \mathrm{keV} \mathrm{X}$-rays and broad band EUV emission unless the time constant for heat conduction from the acceleration region to the lower chromosphere happens to be $\lesssim 10 \mathrm{~s}$. Model 3 removes these difficulties and seems to be consistent with most of the available observations. However, it is considerably more complex than Models 1 and 2. A possible difficulty with Model 3 is the escape of a significant fraction of the energetic $(>20 \mathrm{keV})$ electrons into the lower corona where presumably their energy is slowly dissipated.

On the basis of the available observations above it is not possible at the present time to completely rule out any one of the three models. Observations with higher spatial and time resolution will have to be made before any one of these models can be fully proved or disproved.

\section{Future Work}

Although the problem of the impulsive phase is far from being fully solved, we have arrived at a stage where self-consistent models of emission sources can be constructed in some detail. Several such models have been published in the past two years and hopefully more theoretical work in this direction will be done in the years to come.

Since the problem of the impulsive phase is now fairly well defined, new observations to study specific aspects of the problem are required. For example, the basic time constant of the electron acceleration process can be determined by high time resolution $(\sim 0.1 \mathrm{~s})$ measurements of hard X-ray spectrum. Such measurements with two spacecraft widely separated in heliocentric longitude will help to determine the directivity of hard X-ray emission and the altitude of the hard X-ray source above the photosphere. The question of escape of energetic electrons into the lower corona and their subsequent trapping in the coronal magnetic field can be resolved by a hard X-ray heliograph flown on a suitable satellite.

The measurements of EUV emission with satellites have just begun. Better measurements with higher time resolution $(\$ 1 \mathrm{~s})$ and spacial resolution are required before the energy transport during the impulsive phase can be understood. 
The high resolution $\mathrm{H} \alpha$ measurements now available have helped to remove many uncertainties regarding the relationship between the $\mathrm{H} \alpha$ emission and other impulsive emissions. However the broad band optical measurements are still behind the times, partly due to instrumental difficulties and perhaps partly due to lack of interest. Hopefully this will be rectified soon.

The high resolution microwave measurements have now shown the existence of structure in the microwave source. Hopefully comparable hard X-ray and EUV measurements will be available in the future so that relative locations of the impulsive emission sources can be determined.

It is very satisfying to see measurements in the infra-red (in progress) and milimeter region completing the observed range of electromagnetic emission spectrum from flares. Like white light emission, the detectable infra-red and $\mathrm{mm}$ radiation are apparently associated with relatively large flares. However, these measurements are important for determining the energy balance of a flare.

\section{Acknowledgements}

The author wishes to acknowledge discussions with Drs R. P. Lin, K. A. Anderson, R. F. Donelly, D. L. McKenzie, D. Datlowe, H. S. Hudson, T. Takakura, S. Enome and J. C. Brown. This research was supported in part by the National Aeronautics and Space Administration under grant NGL05-003-017 and NGR-05-003-510. The author's travel to Surfer's Paradise, Australia was partially funded through a travel grant from the National Academy of Sciences.

\section{References}

Acton, L. W.: 1968, Astrophys. J. 152, 305.

Akalane, K.: 1956, Publ. Astron. Soc. Japan 8, 173.

Alfvén, H. and Carlqvist, P.: 1967, Solar Phys. 1, 220.

Anderson, K. A., Kane, S. R., and Lin, R. P.: 1970, 'Fast Electrons from Solar Flares', presented at the International Seminar on the Problem of Cosmic Ray Generation on the Sun, Leningrad, U.S.S.R., December.

Anderson, K. A. and Winckler, J. R.: 1962, J. Geophys. Res. 67, 4103.

Angle, K. L.: 1968, Astron. J. 73, 553.

Arnoldy, R. L., Kane, S. R.; and Winckler, J. R.: 1967, Solar Phys. 2, 171.

Arnoldy, R. L., Kane, S. R., and Winckler, J. R.: 1968a, Astrophys. J. 151, 711.

Arnoldy, R. L., Kane, S. R., and Winckler, J. R.: 1968b, in K. O. Kiepenheuer (ed.), 'Structure and Development of Solar Active Regions', IAU Symp. 35, 490.

Arnoldy, R. L., Kane, S. R., and Winckler, J. R.: 1968c, Tech. Rep. CR-108, Cosmic Ray Group, School of Physics and Astronomy, Univ. of Minnesota, Minneapolis.

Axisa, F., Martres, M. J., Pick, M., and Soru-Escant, I.: 1973, Solar Phys. 29, 163.

Biswas, S. and Radhakrishman, B.: 1973, Solar Phys. 28, 211.

Boischot, A.: 1972, in E. R. Dyer (ed.), Solar Terrestrial Physics/1970, D. Reidel Publ. Co., DordrechtHolland, p. 87.

Brown, J. C.: 1971, Solar Phys. 18, 489.

Brown, J. C.: 1972, Solar Phys. 25, 158.

Brown, J. C.: 1973, this volume, p. 395.

Bruzek, A.: 1964, in W. N. Hess (ed.), AAS-NASA Symposium on the Physics of Solar Flares, NASA SP-50, p. 301 . 
Bruzek, A.: 1967, in J. N. Xanthakis (ed.), Solar Physics, Interscience Publ., New York, p. 399.

Castelli, J. P. and Barron, W. R.: 1969, Bull. Am. Astron. Soc. 1, 274.

Castelli, J. P. and Richards, D. W.: 1971, J. Geophys. Res. 76, 8409.

Chubb, T. A.: 1972, in E. R. Dyer (ed.), Solar Terrestrial Physics/1970, Part I, D. Reidel Publ. Co., Dordrecht-Holland, p. 99.

Chubb, T. A., Kreplin, R. W., and Friedman, H.: 1966, J. Geophys. Res. 71, 3611.

Cline, T. L., Holt, S. S., and Hones, E. W.: 1968, J. Geophys. Res. 73, 434.

Covington, A. E. and Harvey, G. A.: 1961a, Phys. Rev. Letters 6, 51.

Covington, A. E. and Harvey, G. A.: 1961b, Nature 192, 152.

Croom, D. L.: 1973, in R. Ramaty and R. G. Stone (eds.), Proc. Symposium on High Energy Phenomena on the Sun, held at Goddard Space Flight Center, Greenbelt, Maryland, September 28-30 1972, NASA/ GSFC Preprint X-693-73-193, p. 114.

Datlowe, D. and Lin, R. P.: 1973, Solar Phys. 32, 459.

Datlowe, D. and Peterson, L. E.: 1973, 'OSO-7 Observations of Solar X-Ray Bursts from 28 July to 9 August, 1972', preprint.

Davies, K. and Donnelly, R. F.: 1966, J. Geophys. Res. 71, 2843.

De Feiter, L. D.: 1972, Space Sci. Rev. 13, 827.

De Mastus, H. L. and Stover, R.: 1967, Publ. Astron. Soc. Pacific 79, 615.

Dodson, H. W., Hedeman, E. R., and McMath, R. R.: 1956, Astrophys. J. Suppl. $2,241$.

Dodson, H. W. and Hedeman, E. R.: 1968, Solar Phys. 4, 229.

Donnelly, R. F.: 1968, Solar Phys. 5, 123.

Donnelly, R. F.: 1969, Astrophys. J. Letters 158, L165.

Donnelly, R. F.: 1971, Solar Phys. $20,188$.

Donnelly, R. F.: 1973, in R. Ramaty and R. G. Stone (eds.), Proc. Symposium on High Energy Phenomena on the Sun, held at Goddard Space Flight Center, Greenbelt, Maryland, September 28-30, 1972, NASA/ GSFC Preprint X-693-73-193, p. 242.

Donnelly, R. F., Wood, A. T., Jr., and Noyes, R. W.: 1973, Solar Phys. 29, 107.

Doschek, G. A.: 1972, Space Sci. Rev. 13, 765.

Elcan, M. J.: 1973, Bull. Am. Astron. Soc. 5, 340.

Ellison, M. A.: 1947, Monthly Notices Roy. Astron. Soc. 109, 3.

Ellison, M. A.: 1963, Planetary Space Sci. 11, 597.

Elwert, G. and Haug, E.: 1970, Solar Phys. 15, 234.

Enome, S., Kakinuma, T., and Tanaka, H.: 1969, Solar Phys. 6, 428.

Enome, S. and Tanaka, H.: 1971, in R. Howard (ed.), 'Solar Magnetic Fields', IAU Symp. 43, 413.

Fainberg, J.: 1973, this volume, p. 183.

Frost, K. J. : 1964, in W. N. Hess (ed.), AAS-NASA Symposium on the Physics of Solar Flares, NASA SP-50, 139.

Frost, K. J.: 1969, Astrophys. J. Letters 158, L159.

Frost, K. J., and Dennis, B. R. : 1971, Astrophys. J. 165, 655.

Gruber, D. E., Peterson, L. E., and Vette, J. I.: 1973, in R. Ramaty and R. G. Stone (eds.), Proc. Symposium on High Energy Phenomena on the Sun, held at Goddard Space Flight Center, Greenbelt, Maryland, September 28-30 1972, NASA/GSFC Preprint X-693-73-193, p. 147.

Guidice, D. A. and Castelli, J. P.: 1973, in R. Ramaty and R. G. Stone (eds.), Proc. Symposium on High Energy Phenomena on the Sun, held at Goddard Space Flight Center, Greenbelt, Maryland, September 28-30 1972, NASA/GSFC Preprint X-693-73-193, p. 87.

Hall, L. A.: 1971, Solar Phys. 21, 167.

Hall, L. A., and Hintuegger, H. E., 1969, in C. de Jager and Z. Svestka (eds.), 'Solar Flares and Space Research', COSPAR Symp., 81.

Harvey, G. A.: 1964, Astrophys. J. 139, 16.

Harvey, K. L.: 1971, Solar Phys. 16, 423.

Holt, S. S. and Ramaty, R.: 1969, Solar Phys. 8, 119.

Hudson, H. S. : 1972a, Solar Phys. 24, 414.

Hudson, H. S. : 1972b, paper presented at the Seminar on the Generality of Particle Acceleration of Various Scales in Cosmos, Leningrad, August 16-18, 1972.

Hudson, H. S.: 1973, in R. Ramaty and R. G. Stone (eds.), Proc. Symposium on the High Energy Phenomena on the Sun, Goddard Space Flight Center, Greenbelt, Maryland, September 28-30 1972, NASA/GSFC Preprint X-693-73-193, p. 207. 
Hudson, H. S. and Ohki, K.: 1972, Solar Phys. 23, 155.

Hudston, H. S., Peterson, L. E., and Schwartz, D. A.: 1969, Astrophys. J., 157, 389.

Jager, C. de: 1960, in H. K. Bijl (ed.), Space Research I, North Holl. Publ. Co., Amsterdam, p. 628.

Jager, C. de: 1962, Space Sci. Rev. 1, 487.

Jager, C. de: 1964, in H. Odishaw (ed.), Research in Geophysics, Vol. 1, M.I.T. Press, Cambridge, Mass., Chapter 1.

Jager, C. de: 1969, in C. de Jager and Z. Švestka (eds.), 'Solar Flares and Space Research', COSPAR Symp. 1.

Jager, C. de and Kundu, M. R.: 1963, in W. Priestu (ed.), Space Research III, North Holl. Publ. Co., Amsterdam, p. 836.

Kahler, S. W. and Kreplin, R. W.: 1971, Astrophys. J. 168, 531.

Kahler, S. W., Meekins, J. F., Kreplin, R. W., and Boyer, L. S. : 1970, Astrophys. J. $162,293$.

Kakinuma, T., Yamashita, T., and Enome, S.: 1969, Proc. Res. Inst. Atmospherics, Nagoya Univ. Japan 16, 127.

Kane, S. R.: 1969, Astrophys. J. Letters 157, L139.

Kane, S. R.: 1971, Astrophys. J. 170, 587.

Kane, S. R.: 1972a, Space Sci. Rev. 13, 822.

Kane, S. R.: 1972b, Solar Phys. 27, 174.

Kane, S. R.: 1973a, in R. Ramaty and R. G. Stone (eds.), Proc. Symposium on High Energy Phenomena on the Sun, Goddard Space Flight Center, Greenbelt, Maryland, September 28-30, 1972, NASA/GSFC Preprint X-693-73-193, p. 55.

Kane, S. R.: 1973b, paper presented at the 3rd Meeting of the Solar Physics Division of AAS, Las Cruces, New Mexico, Jan. 4-6.

Kane, S. R. and Anderson, K. A.: 1970, Astrophys. J. 162, 1003.

Kane, S. R. and Donnelly, R. F.: 1971, Astrophys. J. 164, 151.

Kane, S. R. and Hudson, H. S.: 1970, Solar Phys. 14, 414.

Kane, S. R., Kahler, S. W., and Kurfess, J. D.: 1972, Solar Phys. 25, 418.

Kane, S. R. and Lin, R. P.: 1972, Solar Phys. 23, 457.

Kane, S. R. and Winckler, J. R.: 1969a, Tech. Rep. CR-134, Cosmic Ray Group, School of Physics and Astronomy, Univ. of Minnesota, Minneapolis.

Kane, S. R. and Winckler. J. R.: 1969b, Solar Phys. 6, 304.

Kelly, P. T. and Rense, W. A.: 1972, Solar Phys. 26, 431.

Kodama, M., Kusunosi, M., and Ogura, K.: 1971, Rep. Ionosphere Space Res. Japan 25, 285.

Korchak, A. A.: 1967, Soviet Astron. AJ, 11, 258.

Kundu, M. R.: 1961, J. Geophys. Res. 66, 4308.

Kundu, M. R.: 1965, Solar Radio Astronomy, Chaper 13, Interscience Publ., New York.

Kundu, M. R.: 1973, in R. Ramaty and R. G. Stone (eds.), Proc. Symposium on High Energy Phenomena on the Sun, held at Goddard Space Flight Center, Greenbelt, Maryland, September 28-30 1972, NASA/ GSFC Preprint X-693-73-193, p. 104.

Lin, R. P.: 1970, 'Acceleration of 10-100 keV Electrons in Solar Flares', presented at Seminar on the Acceleration of Particles in Near-Earth and Interplanetary Space, Galaxy and Metagalaxy, Leningrad, U.S.S.R., July 13-15.

Lin, R. P.: 1973, in R. Ramaty and R. G. Stone (eds.), Proc. Symposium on High Energy Phenomena on the Sun, held at Goddard Space Flight Center, Greenbelt, Maryland, September 28-30 1972, NASA/ GSFC Preprint X-693-73-193, p. 242.

Lin, R. P.: 1974a, Space Sci. Rev. 16, 189.

Lin, R. P.: 1974b, this volume, p. 201.

Lin, R. P. and Hudson, H. S.: 1971, Solar Phys. 17, 412.

Malville, J. M.: 1962, Astrophys. J. 135, 834.

Mandelstam, S. L.: 1972, Acta Physica Academiae Scientiarum Hungaricae 32, 307.

Maxwell, A.: 1965, in C. de Jager (ed.), The Solar Spectrum, D. Reidel Publ. Co., Dordrecht, Holland, p. 342.

McIntosh, P. S. and Donnelly, R. F.: 1972, Solar Phys. 23, 444.

McKenzie, D. L.: 1972, Astrophys. J. 175, 481.

McKenzie, D. L., Datlowe, D., and Peterson, L. E.: 1973, Solar Phys. 28, 175.

McKenzie, D. L. and Peterson, L. E.: 1973, Bull. Am. Astron. Soc. 3, 340.

Moreton, G. E.: 1964, in W. N. Hess (ed.), AAS-NASA Symposium on the Physics of Solar Flares, NASASP 50, p. 209. 
Najita, K. and Orrall, F. Q.: 1970, Solar Phys. 15, 176.

Neupert, W. M.: 1971, Phil. Trans. Roy. Soc. London 270, 143.

Noyes, R. W.: 1973, in R. Ramaty and R. G. Stone (eds.), Proc. Symposium on High Energy Phenomena on the Sun, held at Goddard Space Flight Center, Greenbelt, Maryland, September 28-30, 1972, NASA/GSFC Preprint X-693-73-193, p. 231.

Ohki, K. I.: 1969, Solar Phys. 7, 260.

Parks, G. K. and Winckler, J. R.: 1969, Astrophys. J. Letters 155, L117.

Parks, G. K. and Winckler, J. R.: 1971, Solar Phys. 16, 186.

Peterson, L. E. and Winckler, J. R.: 1959, J. Geophys. Res. 64, 697.

Peterson, L. E., Datlowe, D. W., and McKenzie, D. L.: 1973, in R. Ramaty and R. G. Stone (eds.), Proc. Symposium on the High Energy Phenomena on the Sun, Goddard Space Flight Center, Greenbelt, Maryland, September 28-30 1972, NASA/GSFC Preprint X-693-73-193, p. 132.

Petrosian, V.: 1973, Astrophys. J. 186, 291.

Phillips, K. J. H.: 1973, Observatory 93, 17.

Phillips, K. J. H. and Neupert, W. M.: 1973, Solar Phys. 32, 209.

Pinter, S.: 1969, Solar Phys. 8, 142.

Ramaty, R. and Petrosian, V.: 1972, Astrophys. J. 178, 241.

Santangelo, N., Horstman, H., and Horstman-Moretti, E.: 1973, Solar Phys. 29, 143.

Smith, D. F.: 1973, this volume, p.

Smith, H. J. and Smith, E. v. P.: 1963, Solar Flares, The McMillan Co., New York.

Stewart, R. T.: 1973, this volume, p.

Sturrock, P. A.: 1968, in K. O. Kiepenheuer (ed.), 'Structure and Development of Solar Active Regions', IAU Symp. 35, 471.

Sturrock, P. A.: 1973, this volume, p.

Švestka, Z. : 1969, in C. de Jager and Z. Švestka (eds.), 'Solar Flares and Space Research', COSPAR Symp., 16.

Švestka, Z.: 1970, Solar Phys. 13, 471.

Syrovatskii, S. I. 1969, in C. de Jager and Z. Švestka (eds.), 'Solar Flares and Space Research', COSPAR Symp., 346.

Syrovatskii, S. I. and Shmeleva, O. P.: 1971, Lebedev Institute Preprint 158.

Takakura, T.: 1968, Solar Phys. 6, 133.

Takakura, T.: 1969, in C. de Jager and Z. Svestka (eds.), 'Solar Flares and Space Research', COSPAR Symp., 165.

Takakura, T.: 1971, Solar Phys. 19, 186.

Takakura, T.: 1972, Solar Phys. 26, 151.

Takakura, T.: 1973, in R. Ramaty and R. G. Stone (eds.), Proc. Symposium on the High Energy Phenomena on the Sun, Goddard Space Flight Center, Greenbelt, Maryland, September 28-30, 1972, NASA/GSFC Preprint X-693-73-193, p. 179.

Takakura, T. and Kai, K.: 1966, Publ. Astron. Soc. Japan 18, 57.

Takakura, T., Ohki, K., Shibuya, N., Fujii, M., Matsuoka, M., Miyamoto, S. Nishimura, J., Oda, M., Ogawara, Y., and Ota, S.: 1971, Solar Phys. 16, 454.

Tanaka, H., Kakinuma, T., and Enome, S.: 1967, Proc. Res. Inst. Atmospherics, Nagoya Univ. Japan 14, 23.

Tanaka, H. and Enome, S.: 1971, Solar Phys. 17, 408.

Tanaka, H. and Zirin, H.: 1973, in R. Ramaty and R. G. Stone (eds.), Proc. Symposium on the High Energy Phenomena on the Sun, Goddard Space Flight Center, Greenbelt, Maryland, September 28-30, 1972, NASA/GSFC Preprint X-693-73-193, p. 26.

Teske, R. G., Soyumer, T., and Hudson, H. S.: 1971, Astrophys. J. 165, 615.

Tindo, I. P., Ivanov, V. D., Mandelstam, S. L., and Shuryghin, A. I.: 1970, Solar Phys. 14, 204.

Tindo, I. P., Ivanov, V. D., Mandelstam, S. L., and Shyryghin, A. I.: 1972a, Solar Phys. 24, 429.

Tindo, I. P., Ivanov, V. D., Valnicek, B., and Livshitz, M. A.: 1972b, Solar Phys. 27, 426.

Tomblin, F. F.: 1972, Astrophys. 171, 377.

Vette, J. I. and Casal, F. G.: 1961, Phys. Rev. Letters 6, 334.

Vorpahl, J. A.: 1972, Solar Phys. 26, 397.

Wild, J. P., Smerd, S. F., and Weiss, A. A.: 1963, Ann. Rev. Astron. Astrophys. 1, 291.

Winckler, J. R., May, T. C., and Masley, A. J.: 1961, J. Geophys. Res. 66, 316.

Wood, A. T., Jr. and Noyes, R. W.: 1972, Solar Phys. 24, 180. 
Wood, A. T., Jr., Noyes, R. W., Dupree, A. K., Huber, M. C. E., Parkinson, W. H., Reeves, E. M., and Withbroe, G. L. : 1972, Solar Phys. 24, 169,

Zirin, H.: 1972, Solar Phys. 26, 393.

Zirin, H.: 1973, 'Solar Flares', review paper for Vistas in Astronomy 16, 1.

Zirin, H., Pruss, G., and Vorpahl, J. : 1971, Solar Phys. 19, 463.

\section{DISCUSSION}

Brown: I would like to disassociate my name from your third schematic flare model. There seems to be a misunderstanding since, although I have studied trapped electron models for the hard X-ray source, I have always advocated a thick target geometry if electrons are to heat the optical and EUV flare. Secondly, it is worth adding that Jerry Drake has shown that the only statistically significant amount of data on hard X-ray burst longitude distribution is in the $1-6 \mathrm{keV}$ band. He found this to be isotropic which it should be since it is mostly thermal.

Kane: Higher energy bursts should be used for such studies. Petrosian has predicted that the X-ray spectra should be harder at the limb. This arises because of the directivity of the high energy bremsstrahlung.

Zirin: There is an empirical way of determining the height of an X-ray burst based on $\mathrm{H} \alpha$ data and a cut-off in the microwave spectrum at $3000 \mathrm{MHz}$. These data indicate a low height.

Kane: This may happen in some events but the lack of center-to-limb variation in the microwave radiation indicates that the source is high.

Zirin: Those results may not be trustworthy.

Smith: There are several mechanisms for the microwave cut-off. With our present knowledge of a flare, selection of one particular michanism over the others, namely absorption below the plasma frequency, is not necessarily valid.

Zirin: I have looked at free-free absorption, Razin effect and others and they all imply a low source height.

Takakura: Self absorption could produce a cut-off for a high source if the source were small and the number of nonthermal electrons were high, say $10^{3}-10^{4} \mathrm{~cm}^{-3}$ above $100 \mathrm{keV}$.

Zirin: Wouldn't this produce visible absorption?

Brueckner: How do you obtain the number $10^{28} \mathrm{erg}$ in the UV for a major flare?

Kane: From SFD data which has now been calibrated by spacecraft observations in the EUV.

Kundu: Have you any data available on the X-ray spectrum of bursts associated with gradual rise and fall centimeter burst?

Kane: Soft X-rays - we don't have much data but should expect the gradual rise and fall to have a thermal spectrum.

Kundu: That is precisely the reason I am asking. Recent high resolution interferometric observations with resolution of seconds of arc of a gradual rise and fall type $\mathrm{cm}$ burst indicates that a significant fraction of the burst energy is emitted within a volume of $2^{\prime \prime}$ diam. The brightness temperature that we get is about $10^{9} \mathrm{~K}$. Consequently, this type of $\mathrm{cm}$ burst, which was previously thought to be thermal, is certainly nonthermal. I am just wondering if you have any nonthermal component of X-rays associated with such bursts.

Kane: The X-ray component of the gradual rise and fall may be too weak to have been measured yet.

Sturrock: Could you comment further on the emission process in the flash phase; is it thin or thick target?

Kane: Since the ion density in (or altitude of) the X-ray source is not known, it is not possible at present to rule out either the thick- or thin-target models. In the few events where simultaneous X-ray and interplanetary electron observations have been compared, the thin target model fits the observations better. But the question is not resolved.

Brown: Your comparison of interplanetary electron spectra and those inferred from hard X-rays unfortunately does not settle the thick-thin target controversy. The problem is that the interplanetary electrons comprise only $0.1 \%$ (Lin and Datlowe, 1973) of those in the X-ray source and are therefore probably most uncharacteristic of the latter. Thus, if more energetic electrons escaped preferentially, the spectral evidence would favor a thick rather than thin target. 